\title{
Unilateral sources and sinks of an activator in reaction-diffusion systems exhibiting diffusion-driven instability
}

\author{
Martin Fencl ${ }^{\mathrm{a}}$, Milan Kučera ${ }^{\mathrm{a}, \mathrm{b}}$ \\ ${ }^{a}$ Dept. of Mathematics, Faculty of Applied Sciences, University of West Bohemia in Pilsen, Univerzitni 8, 30614 \\ Plzeñ, Czech Republic \\ ${ }^{b}$ Institute of Mathematics, Czech Academy of Sciences, Žitná 25, 11567 Prague 1, Czech Republic
}

\begin{abstract}
A reaction-diffusion system exhibiting Turing's diffusion driven instability is considered. The equation for an activator is supplemented by unilateral terms of the type $s_{-}(\mathbf{x}) u^{-}, s_{+}(\mathbf{x}) u^{+}$describing sources and sinks active only if the concentration decreases below and increases above, respectively, the value of the basic spatially constant solution which is shifted to zero. We show that the domain of diffusion parameters in which spatially non-homogeneous stationary solutions can bifurcate from that constant solution is smaller than in the classical case without unilateral terms. It is a dual information to previous results stating that analogous terms in the equation for an inhibitor imply the existence of bifurcation points even in diffusion parameters for which bifurcation is excluded without unilateral sources. The case of mixed (Dirichlet-Neumann) boundary conditions as well as that of pure Neumann conditions is described.
\end{abstract}

Keywords: reaction-diffusion systems, unilateral terms, Turing's patterns, positively homogeneous operators, maximal eigenvalue

MSC: $35 \mathrm{~K} 57,35 \mathrm{~B} 32,35 \mathrm{~J} 57,35 \mathrm{~J} 50,92 \mathrm{C} 15$

\section{Introduction}

Let's consider a reaction-diffusion system

$$
\begin{aligned}
& \frac{\partial u}{\partial t}=d_{1} \Delta u+f(u, v)+\tilde{f}_{-}\left(\mathbf{x}, u^{-}\right)-\tilde{f}_{+}\left(\mathbf{x}, u^{+}\right), \\
& \frac{\partial v}{\partial t}=d_{2} \Delta v+g(u, v) \quad \text { in } \Omega \times[0,+\infty)
\end{aligned}
$$

where $\Omega \subset \mathbb{R}^{N}$ is a bounded domain with Lipschitz boundary, $d_{1}$ and $d_{2}$ are positive parameters (diffusion coefficients), $f, g: \mathbb{R} \times \mathbb{R} \rightarrow \mathbb{R}$ are real differentiable functions, $\tilde{f}_{-}, \tilde{f}_{+}: \Omega \times \mathbb{R} \rightarrow \mathbb{R}$ are functions satisfying Carathéodory conditions and such that there exist

$$
s_{-}(\mathbf{x}):=\left.\frac{\partial \tilde{f}_{-}}{\partial \xi}(\mathbf{x}, \xi)\right|_{\xi=0} \geq 0, \quad s_{+}(\mathbf{x}):=\left.\frac{\partial \tilde{f}_{+}}{\partial \xi}(\mathbf{x}, \xi)\right|_{\xi=0} \geq 0 \quad \text { for a.a. } \mathbf{x} \in \Omega, s_{ \pm} \in L_{\infty}(\Omega) .
$$

As usually, $u^{+}=\max \{u, 0\}$ and $u^{-}=\max \{-u, 0\}$ denotes the positive and negative, respectively, part of $u$. We will always assume that

$$
f(0,0)=g(0,0)=\tilde{f}_{-}(\mathbf{x}, 0)=\tilde{f}_{+}(\mathbf{x}, 0)=0 \quad \text { for a.a. } \mathbf{x} \in \Omega .
$$

Our system will be supplemented by boundary conditions

$$
\begin{aligned}
& u=v=0 \quad \text { on } \Gamma_{D}, \\
& \frac{\partial u}{\partial n}=\frac{\partial v}{\partial n}=0 \quad \text { on } \Gamma_{N},
\end{aligned}
$$

Email addresses: fenclm37@kma.zcu.cz (Martin Fencl), kucera@math.cas.cz (Milan Kučera) 
where $n$ is the unit outward-pointing normal vector of the boundary $\partial \Omega$ and $\Gamma_{N}, \Gamma_{D}$ are open disjoint subsets of $\partial \Omega, \partial \Omega=\overline{\Gamma_{D}} \cup \overline{\Gamma_{N}}$.

Apparently the problem (1), (4) has always the trivial solution $[0,0]$. Our system should describe a reaction of two chemicals, e.g. morphogens, having a basic positive spatially constant steady state $[\bar{u}, \bar{v}]$, that means we should assume in fact $f(\bar{u}, \bar{v})=g(\bar{v}, \bar{v})=\tilde{f}_{-}(\mathbf{x}, \bar{u})=\tilde{f}_{+}(\mathbf{x}, \bar{u})=0$ instead of (3), but as usually, we can shift the positive steady state to zero and we obtain our system satisfying (3). Let us emphasize that then the functions $u, v$ do not describe concentrations of the reactants, but their differences from the basic constant stationary state $[\bar{u}, \bar{v}]$.

We will consider assumptions under which the problem (1), (4) with $\tilde{f}_{-} \equiv \tilde{f}_{+} \equiv 0$ exhibits diffusion driven instability discovered in the famous Turing's paper [11]. That means if $\tilde{f}_{-} \equiv \tilde{f}_{+} \equiv 0$ then the trivial solution $[0,0]$ is stable as a solution of the corresponding problem without diffusion (ODE's obtained for $d_{1}=d_{2}=0$ ), but as a solution of the whole system it is unstable for $\left[d_{1}, d_{2}\right]$ from a certain subdomain $D_{U}$ of the positive quadrant $\mathbb{R}_{+}^{2}$ (domain of instability), and stable only for $\left[d_{1}, d_{2}\right] \in D_{S}=\mathbb{R}_{+}^{2} \backslash \overline{D_{U}}$ (domain of stability). Spatially non-homogeneous steady states bifurcate from the basic constant equilibrium in some points of $\overline{D_{U}}$, but such a bifurcation is excluded in $D_{S}$. Let us note that spatially non-homogeneous steady states can describe spatial patterns in some models in biology.

Our goal is to prove that if we add unilateral terms $\tilde{f}_{-}\left(\mathbf{x}, u^{-}\right), \tilde{f}_{+}\left(\mathbf{x}, u^{+}\right)$, then the domain of diffusion coefficients where spatially non-homogeneous steady states can bifurcate is smaller than $\overline{D_{U}}$. In fact we will prove more, see below. An example of unilateral terms can be

$$
\tilde{f}_{-}\left(\mathbf{x}, u^{-}\right)=s_{-}(\mathbf{x}) \frac{u^{-}}{1+\varepsilon u^{-}}, \quad \tilde{f}_{+}\left(\mathbf{x}, u^{+}\right)=s_{+}(\mathbf{x}) \frac{u^{+}}{1+\varepsilon u^{+}} .
$$

The stationary system corresponding to (1) can be written in the form

$$
\begin{aligned}
d_{1} \Delta u+b_{1,1} u+b_{1,2} v+n_{1}(u, v)+\tilde{f}_{-}\left(\mathbf{x}, u^{-}\right)-\tilde{f}_{+}\left(\mathbf{x}, u^{+}\right) & =0 \\
d_{2} \Delta v+b_{2,1} u+b_{2,2} v+n_{2}(u, v) & =0
\end{aligned}
$$

where $\mathbf{B}:=\left(b_{i, j}\right)_{i, j=1,2}$ is the Jacobi matrix of the mappings $f, g$ at $[0,0]$ and the functions $n_{1}, n_{2}$ are higher order terms, i.e.

$$
n_{1,2}(u, v)=o(|u|+|v|) \text { as }|u|+|v| \rightarrow 0 .
$$

(The nonlinear part in the first equation could be written also in the form $s_{-}(\mathbf{x}) u^{-}-s_{+}(\mathbf{x}) u^{+}+$ $\tilde{n}_{1}(\mathbf{x}, u, v)$, that means a homogenization + higher order terms dependent on $\left.\mathbf{x}\right)$.

We will always assume that the following conditions necessary for Turing's diffusion driven instability mentioned above are fulfilled:

$$
b_{1,1}>0, b_{2,2}<0, b_{1,2} b_{2,1}<0, \operatorname{tr}(\mathbf{B})<0, \operatorname{det}(\mathbf{B})>0 .
$$

The first three conditions in (7) correspond to an activator-inhibitor system (for $b_{1,2}<0, b_{2,1}>0$ ), or to a substrate depletion system (for $b_{1,2}>0, b_{2,1}<0$ ), see e.g. [9]. The last two conditions ensure the stability of $[0,0]$ as a solution of the system without any diffusion.

We will work mainly with the homogenized system

$$
\begin{aligned}
d_{1} \Delta u+b_{1,1} u+b_{1,2} v+s_{-}(\mathbf{x}) u^{-}-s_{+}(\mathbf{x}) u^{+} & =0, \\
d_{2} \Delta v+b_{2,1} u+b_{2,2} v & =0 .
\end{aligned}
$$

We will show more than what is mentioned above, namely that critical points, i.e. couples $\left[d_{1}, d_{2}\right]$ for which the homogenized problem (8), (4) has a non-trivial solution, can exist only in a smaller domain than in the classical case $\tilde{s}_{-}=\tilde{s}_{+} \equiv 0$. Since any bifurcation point is simultaneously a critical point, the main goal mentioned above will follow. A similar result was proved in [5] for the case of unilateral sources on the boundary described by quasi-variational inequalities, but we consider the description of unilateral sources and sinks by the terms $\tilde{f}_{-}\left(\mathbf{x}, u^{-}\right), \tilde{f}_{+}\left(\mathbf{x}, u^{+}\right)$more 
natural. We will briefly discuss also problems with unilateral terms of the type $s_{-}(x) u^{-}, s_{+}(x) u^{+}$ on the boundary.

Main ideas are similar to those from [5]. Considering a weak formulation, we will write our problem as a system of operator equations in Sobolev space and we will consider an arbitrary fixed $d_{2}$. Expressing the variable $v$ from the second equation and substituting it to the first equation, we reduce the originally non-symmetric problem to a single equation with a positively homogeneous operator having a potential. A variational characterization of its largest eigenvalue enables us to compare the largest eigenvalue corresponding to the problem with and without unilateral terms, which is simultaneously the largest $d_{1}$ for which $\left[d_{1}, d_{2}\right]$ is a critical point of the original system with and without unilateral terms.

Let us note that if unilateral sources of the second variable $v$ (inhibitor) are supplemented in the second equation then bifurcation of spatial patterns occurs even in the domain $D_{S}$, where it is excluded for the classical case without unilateral sources. See e.g. [7] and references therein for the case of sources described by variational inequalities, [4] for unilateral sources described by multivalued maps and [3], [6] for the case of unilateral terms similar to the current paper. These results motivated numerical experiments [12] showing that for a concrete model also spatial patterns arise from small initial perturbations for diffusion parameters from $D_{S}$, where it is not the case without unilateral sources. The sense of these results is positive because one of the problems of Turing's theory is that the set of diffusion parameters for which diffusion-driven instability occurs is too small, so unilateral sources for $v$ improve this situation. The result of the current paper is opposite, unilateral sources for $u$ makes larger the set of diffusion parameters for which bifurcation of spatial patterns is exluded, i.e. for which no small spatial patterns can exist. We believe that, at least in some cases, it is a signal that the same is true for the set of parameters for which spatial patterns evolve from small perturbations of the basic spatially constant steady state. It agrees with numerical experiments which will be published in a forthcoming paper. This seems to be a negative result, but perhaps there are situations when it would be valuable to understand how to prevent evolution of spatial patterns. For instance, patterns play a role in models of tumors, see e.g. [1] and references therein. In spite of that the paper [1] has completely different goals, it can be perhaps motivating from the point of view mentioned, in particular its Section 5 .

We present the basic general assumptions and definitions in Section 2. Main results of this paper are formulated and discussed in Section 3. In Section 4 we formulate our problem as a system of operator equations in Sobolev space and we describe properties of the corresponding operators. Section 5 concerns a reduction of our system to a single equation with a positively homogeneous operator and a variational characterization of its largest eigenvalue. A comparison of largest eigenvalues and consequently also critical points with and without unilateral terms by using this variational characterization is given. The proofs of the main results are done in Section 6.

\section{Basic assumptions and definitions}

We will always suppose that there exists $c \in \mathbb{R}$ such that

$$
\begin{array}{r}
\left|n_{j}(\chi, \xi)\right| \leq c\left(1+|\chi|^{q-1}+|\xi|^{q-1}\right) \quad \text { for all } \chi, \xi \in \mathbb{R}, j=1,2, \\
\left|\tilde{f}_{\mp}(\mathbf{x}, \xi)\right| \leq c\left(1+|\xi|^{q-1}\right) \quad \text { for all } \xi \in \mathbb{R} \text { and a.a. } \mathbf{x} \in \Omega,
\end{array}
$$

with some $q>2$ if $N=2$ or $2<q<\frac{2 N}{N-2}$ if $N>2$. In the dimension $N=1$ no growth assumptions are necessary.

Besides systems (8) and (5) we will discuss systems

$$
\begin{array}{r}
d_{1} \Delta u+b_{1,1} u+b_{1,2} v=0, \\
d_{2} \Delta v+b_{2,1} u+b_{2,2} v=0
\end{array}
$$

and

$$
\begin{aligned}
& d_{1} \Delta u+b_{1,1} u+b_{1,2} v+n_{1}(u, v)=0, \\
& d_{2} \Delta v+b_{2,1} u+b_{2,2} v+n_{2}(u, v)=0 .
\end{aligned}
$$


By solutions we will always mean weak solutions in the space

$$
H_{D}^{1}(\Omega):=\left\{\phi \in W^{1,2}(\Omega): \phi=0 \text { on } \Gamma_{D} \text { in the sense of traces }\right\} .
$$

If $\Gamma_{D}=\emptyset$, then the space $H_{D}^{1}$ is actually the whole Sobolev space $W^{1,2}$ equipped with the standard inner product

$$
(u, \varphi)_{H_{D}^{1}}=(u, \varphi)_{W^{1,2}}=\int_{\Omega}(\nabla u \nabla \varphi+u \varphi) d \Omega
$$

and the Sobolev norm $\|u\|_{W^{1,2}}=\left(\int_{\Omega}(\nabla u)^{2}+u^{2} d \Omega\right)^{\frac{1}{2}}$. If $\Gamma_{D} \neq \emptyset$, then we will use the inner product

$$
(u, \varphi)_{H_{D}^{1}}=\int_{\Omega} \nabla u \nabla \varphi d \Omega
$$

and the norm $\|u\|_{H_{D}^{1}}=\left(\int_{\Omega}(\nabla u)^{2} d \Omega\right)^{\frac{1}{2}}$ equivalent to the classical Sobolev norm.

Definition 2.1 (Critical point).

A parameter $d=\left[d_{1}, d_{2}\right] \in \mathbb{R}_{+}^{2}$ will be called a critical point of (11), (4) or (8), (4) if there exists a non-trivial (weak) solution of (11), (4) or (8), (4), respectively.

Definition 2.2 (Bifurcation point).

A parameter $d^{0}=\left[d_{1}^{0}, d_{2}^{0}\right] \in \mathbb{R}_{+}^{2}$ will be called a bifurcation point of (12), (4) or (5), (4) if in any neighbourhood of $\left[d^{0}, 0,0\right] \in \mathbb{R}_{+}^{2} \times H_{D}^{1} \times H_{D}^{1}$ there exists $[d, W]=[d, u, v],\|W\| \neq 0$ satisfying (12), (4) or (5), (4), respectively.

Remark 2.1. Let's consider the problem

$$
\begin{aligned}
-\Delta u & =\kappa u, \\
u & =0 \text { on } \Gamma_{D}, \\
\frac{\partial u}{\partial n} & =0 \text { on } \Gamma_{N} .
\end{aligned}
$$

The eigenvalues of (16) form a non-negative non-decreasing sequence $\kappa_{j}$ with $j=1,2, \ldots$ (for $\Gamma_{D} \neq \emptyset$ ) or $j=0,1,2, \ldots$ (for $\Gamma_{D}=\emptyset$ ). The first eigenvalue is always simple. In the case $\Gamma_{D} \neq \emptyset$, the eigenfunction $e_{1}$ corresponding to the first eigenvalue $\kappa_{1}$ does not change the sign on the domain $\Omega$. In the case $\Gamma_{D}=\emptyset$, the eigenfunction $e_{0}$ corresponding to the first eigenvalue $\kappa_{0}=0$ is constant. Other eigenfunctions change the sign in both cases. We can choose an orthonormal basis $e_{j}$ in $H_{D}^{1}, j=1,2, \ldots\left(\right.$ for $\left.\Gamma_{D} \neq \emptyset\right)$ or $j=0,1,2, \ldots\left(\right.$ for $\left.\Gamma_{D}=\emptyset\right)$ composed of the eigenfunctions of (16).

Let's remind that the conditions (7) are always considered. The sets

$$
C_{j}:=\left\{\left[d_{1}, d_{2}\right] \in \mathbb{R}_{+}^{2}: d_{1}=\frac{1}{\kappa_{j}}\left(\frac{b_{1,2} b_{2,1}}{d_{2} \kappa_{j}-b_{2,2}}+b_{1,1}\right)\right\}, j=1,2, \ldots
$$

are hyperbolas (or more specifically their parts) in the positive quadrant $\mathbb{R}_{+}^{2}$. Let's note that we present hyperbolas in the different form than usually, namely with respect to $d_{1}$. It is of course equivalent to the standard form derived from the relation

$$
\left(\kappa_{j} d_{1}-b_{1,1}\right)\left(\kappa_{j} d_{2}-b_{2,2}\right)-b_{1,2} b_{2,1}=0
$$

(see e.g. [9]). If $\Gamma_{D}=\emptyset$, for $j=0$ the last equality is never satisfied, because $\operatorname{det}(B)$ is positive by (7). The envelope

$$
C_{E}:=\left\{d=\left[d_{1}, d_{2}\right] \in \mathbb{R}_{+}^{2}: d_{1}=\max _{\tilde{d}_{1} \in \mathbb{R}_{+}}\left\{\tilde{d}_{1}:\left[\tilde{d}_{1}, d_{2}\right] \in \bigcup_{j=1}^{\infty} C_{j}\right\}\right\}
$$

divides the positive quadrant $\mathbb{R}_{+}^{2}$ onto two sets $D_{U}$ and $D_{S}$ (see Figure 1 ). 


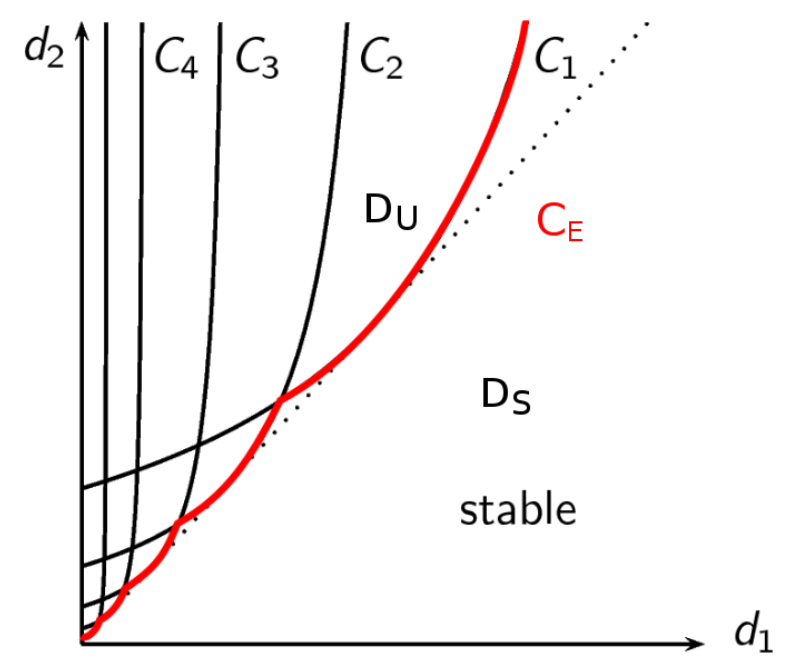

Figure 1: Illustration of the hyperbolas $C_{j}$ and the envelope $C_{E}$. The case when all eigenvalues $\kappa_{j}$ are simple.

Remark 2.2. If all eigenvalues of (16) are simple, i.e. $\kappa_{j}<\kappa_{j+1}$ for all $j \in \mathbb{N}$, then $C_{j} \neq C_{j+1}$ for all $j>0$. If an eigenvalue $\kappa_{j}$ has a multiplicity $k$, then $\kappa_{j-1}<\kappa_{j}=\ldots=\kappa_{j+k-1}<\kappa_{j+k}$ and $C_{j-1} \neq C_{j}=\ldots=C_{j+k-1} \neq C_{j+k}$. The sets

$$
\begin{gathered}
D_{U}:=\left\{d=\left[d_{1}, d_{2}\right] \in \mathbb{R}_{+}^{2}: d \text { is on the left of } C_{E}\right\}, \\
D_{S}:=\left\{d=\left[d_{1}, d_{2}\right] \in \mathbb{R}_{+}^{2}: d \text { is on the right of } C_{E}\right\}
\end{gathered}
$$

are called the domain of instability and the domain of stability. It is known that if $\left[d_{1}, d_{2}\right] \in D_{S}$, then all eigenvalues $\lambda$ of the problem deciding about stability of the trivial solution of the evolution system corresponding to (12), (4) have negative real parts and if $\left[d_{1}, d_{2}\right] \in D_{U}$, then there is an eigenvalue $\lambda$ with positive real part (for a particular case see [8],[10] and for a general case [2]). In particular, the trivial solution of $(12),(4)$ is linearly stable for $\left[d_{1}, d_{2}\right] \in D_{S}$ and unstable for $\left[d_{1}, d_{2}\right] \in D_{U}$.

Remark 2.3. The following properties of the curves $C_{j}$ are known, see e.g. [10],[8] for a particular case, or [2] for the general case.

- A point $d=\left[d_{1}, d_{2}\right]$ is a critical point of $(11),(4)$ if and only if there exists $j$ such that $d \in C_{j}$. In particular, the domain of stability $D_{S}$ does not contain any critical point of (11), (4) or bifurcation point of (12),(4). Under some additional assumptions, e.g. if the eigenvalue $\kappa_{j}$ is simple or of odd multiplicity, the points on $C_{j}$ are simultaneously bifurcation points (see e.g. [10]).

- If $d \in C_{n}$ for $n=j, \ldots, j+k-1$ (either $k$ is the multiplicity of the eigenvalue $\kappa_{j}$ or $d$ is in the intersection of two hyperbolas $C_{j}, C_{m}$ and $k$ is the sum of multiplicities of $\kappa_{j}, \kappa_{m}$, see Remark 2.2), then span $\left(\left[\frac{d_{2} \kappa_{j}-b_{2,2}}{b_{2,1}} e_{j}, e_{j}\right]_{n=j}^{j+k-1}\right)$ is the set of the solutions of (11), (4).

\section{Main results}

Let's recall that the assumptions (9),(10) are automatically supposed. Besides the notions introduced in Section 2 we will use the following symbols.

\section{Notation 3.1.}

Let $r, R, \varepsilon \in \mathbb{R}_{+}$and $r<R$. We define

$C_{r}^{R}:=\left\{d=\left[d_{1}, d_{2}\right] \in C_{E}: d_{2} \in[r, R]\right\}$

$C_{r}^{R}(\varepsilon):=\left\{d=\left[d_{1}, d_{2}\right] \in C_{E} \cup D_{U}: d_{2} \in[r, R] \wedge \operatorname{dist}\left(d, C_{E}\right)<\varepsilon\right\}$. 
The following theorem is the main result of this paper.

Theorem 3.1. $\quad$ i) The domain of stability $D_{S}$ contains neither critical points of (8),(4) nor bifurcation points of (5), (4).

ii) Let $0<r<R$. Let $C_{j}, \ldots, C_{j+k-1}$ be all hyperbolas which have a non-empty intersection with $C_{r}^{R}$. Let any linear combination e of the eigenfunctions of (16) corresponding to $\kappa_{j}, \ldots, \kappa_{j+k-1}$ satisfy

$$
s_{-} e^{-}-s_{+} e^{+} \not \equiv 0 .
$$

Then there exists $\varepsilon>0$ such that there are neither critical points of (8), (4) nor bifurcation points of (5), (4) in $C_{r}^{R}(\varepsilon)$.

We emphasize that if the condition (19) is not satisfied for some linear combination $e$ mentioned, then there are critical points of (8),(4) directly on $C_{r}^{R}$ due to Remark 2.3. Let's note that if all hyperbolas $C_{j}, \ldots, C_{j+k-1}$ do not coincide, i.e. it is not $\kappa_{j}=\kappa_{j+1}=\ldots=\kappa_{j+k-1}$, then the eigenfunctions $e_{j}, \ldots, e_{j+k-1}$ do not correspond to the same eigenvalue and their linear combination need not be an eigenfunction. We discuss possible situations in the following two examples:

- First let's assume that $C_{r}^{R}$ has a non-empty intersection with exactly two non-coinciding hyperbolas $C_{k}$ and $C_{k+1}$. If both $e=e_{k}$ and $e=e_{k+1}$ satisfy (19), then there are no critical points of (8), (4) on $C_{r}^{R} \backslash\left(C_{k} \cap C_{k+1}\right)$. However, it can happen that there is a linear combination $e$ of $e_{k}, e_{k+1}$ such that $s_{-} e^{-}-s_{+} e^{+} \equiv 0$, and in this case the intersection point $C_{k} \cap C_{k+1}$ is a critical point of (8), (4) (see also Remark 2.3).

- In an other scenario we take $C_{r}^{R}$ which consists of a part of two coinciding hyperbolas $C_{k}=C_{k+1}$, i.e. $\kappa_{k}=\kappa_{k+1}$. In this case the assumption of Theorem 3.1 ii) means that every eigenfunction corresponding to $\kappa_{k}=\kappa_{k+1}$ must satisfy (19). Otherwise the critical points of (8), (4) are on the whole $C_{k}$, in particular on $C_{r}^{R}$ (see Remark 2.3).

The result is illustrated on Figure 2.

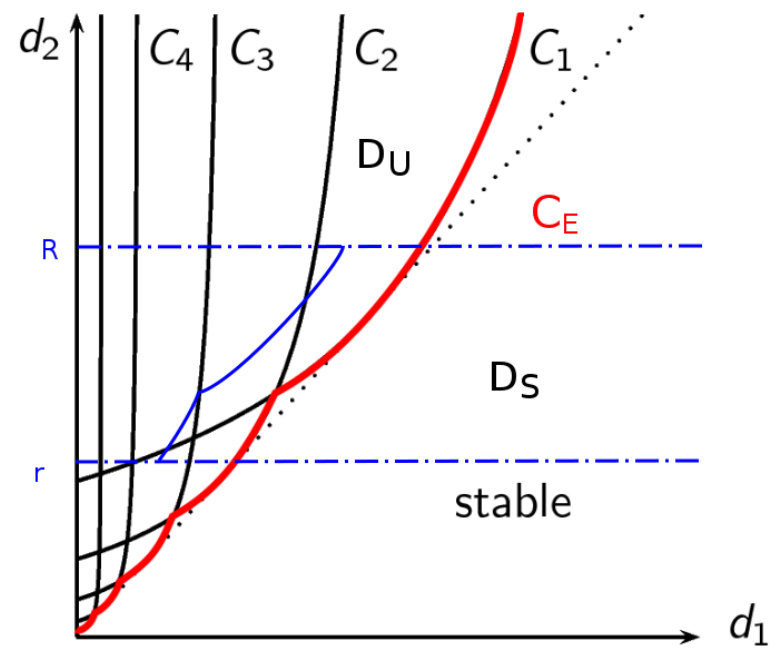

Figure 2: Illustration of the result of Theorem 3.1. The critical points are no longer in the region between $C_{E}$ (red curve) and blue curve. Assuming the case when all eigenvalues $\kappa_{j}$ are simple, i.e. $C_{j} \neq C_{k}$ for all $k \neq j$, and any linear combination of eigenfunctions $e_{1}, e_{2}$ corresponding to $\kappa_{1}, \kappa_{2}$ satisfy (19).

Corollary 3.1. $\quad$ i) For any compact part $M$ of $D_{S}$ there exists $\delta>0$ such that for any $\left[d_{1}, d_{2}\right] \in$ $M$ there are no non-trivial solutions of (5),(4) with $0<\|u\|_{H_{D}^{1}}+\|v\|_{H_{D}^{1}}<\delta$. 
ii) Under the assumption from Theorem 3.1 ii), for any compact part $M$ of $D_{S} \cup C_{r}^{R}(\varepsilon)$ there exists $\delta>0$ such that for any $\left[d_{1}, d_{2}\right] \in M$ there are no non-trivial solutions of (5), (4) with $0<\|u\|_{H_{D}^{1}}+\|v\|_{H_{D}^{1}}<\delta$.

Proof.

Indeed, it is easy to see that if this were not true, then a bifurcation point of (5), (4) would exist in $M$, which would contradict Theorem 3.1.

There are two important particular cases for $\Gamma_{D} \neq \emptyset$ and $\Gamma_{D}=\emptyset$ :

Theorem 3.2. Let $\Gamma_{D} \neq \emptyset$. Let one of the functions $s_{+}, s_{-}$be identically zero and the other positive a.e. on $\Omega$. Let $d_{2}^{I}$ be the second coordinate of the intersection point of $C_{1}$ and $C_{2}$.

i) Any $d \in C_{1}$, in particular any $d \in C_{r}^{R}$ with $d_{2}^{I} \leq r<R$, is a critical point of (8),(4).

ii) If $0<r<R<d_{2}^{I}$, then there exists $\varepsilon>0$ such that there are neither critical points of (8),(4) nor bifurcation points of (5), (4) in $C_{r}^{R}(\varepsilon)$.

Theorem 3.3. Let $\Gamma_{D}=\emptyset$. Let one of the functions $s_{+}, s_{-}$be identically zero and the other positive a.e. on $\Omega$. Then for any $0<r<R$ there exists $\varepsilon>0$ such that there are neither critical points of (8),(4) nor bifurcation points of (5), (4) in $C_{r}^{R}(\varepsilon)$.

Remark 3.1. The size of $\varepsilon$ in Theorems 3.1-3.3 depends on $r$ and $R$. Actually $\varepsilon \rightarrow 0$ as $R \rightarrow d_{2}^{I}$ or $r \rightarrow 0$ in Theorem 3.2 and $\varepsilon \rightarrow 0$ as $r \rightarrow 0$ in Theorem 3.3. The following theorem states that if the source and sink are in some sense small enough, then there exists at least one critical point $\left[d_{1}, d_{2}\right] \in D_{U} \cup C_{E}$ with a given $d_{2}$. A question if sometimes (for a strong source or sink) no critical point with a given $d_{2}$ exists remains an open problem. Cf. Remark 5.5 in Section 5.

Theorem 3.4. Let $d_{2}>0$ be arbitrary fixed. Let $j_{0}$ be such that $\left[\frac{1}{\kappa_{j_{0}}}\left(\frac{b_{1,2} b_{2,1}}{d_{2} \kappa_{j_{0}}-b_{2,2}}+b_{1,1}\right), d_{2}\right] \in C_{E}$ (see (17),(18)). If $\max \left\{\left\|s_{-}\right\|_{\infty},\left\|s_{+}\right\|_{\infty}\right\}<b_{1,1}+\frac{b_{1,2} b_{2,1}}{d_{2} \kappa_{j_{0}}-b_{2,2}}$, then there exists at least one $d_{1}$ such that $\left[d_{1}, d_{2}\right] \in D_{U} \cup C_{E}$ is a critical point of the problem (8), (4).

The last theorem of this section is a modification of Theorem 3.1 for the case of unilateral terms in boundary conditions, namely for systems (11) and (12) with boundary conditions

$$
\begin{aligned}
& u=v=0 \quad \text { on } \quad \Gamma_{D}, \\
& \frac{\partial u}{\partial n}=s_{-}(\mathbf{x}) u^{-}-s_{+}(\mathbf{x}) u^{+} \quad \text { on } \quad \Gamma_{N}, \\
& \frac{\partial v}{\partial n}=0 \quad \text { on } \quad \Gamma_{N} .
\end{aligned}
$$

Let us note that we consider only positively homogeneous boundary conditions because introducing more general boundary terms as $\tilde{f}_{\mp}$ in the case of sources and sinks in the interior of the domain would mean additional technical complications.

Theorem 3.5. $\quad$ i) The domain of stability $D_{S}$ contains neither critical points of (11),(20) nor bifurcation points of (12), (20).

ii) Let $0<r<R$. Let $C_{j}, \ldots, C_{j+k-1}$ be all hyperbolas which have a non-empty intersection with $C_{r}^{R}$. Let any linear combination e of the eigenfunctions of (16) corresponding to $\kappa_{j}, \ldots, \kappa_{j+k-1}$ satisfy

$$
s_{-} e^{-}-s_{+} e^{+} \not \equiv 0 \quad \text { on } \Gamma_{N} .
$$

Then there exists $\varepsilon>0$ such that there are neither critical points of (11),(20) nor bifurcation points of (12), (20) in $C_{r}^{R}(\varepsilon)$.

Analogous consequence as in Corollary 3.1 can be formulated for Theorems 3.2, 3.3 and 3.5. 


\section{Abstract formulation}

We define the operator $A: H_{D}^{1} \mapsto H_{D}^{1}$ as

$$
(A u, \varphi)=\int_{\Omega} u \varphi d \Omega \quad \text { for all } u, \varphi \in H_{D}^{1}(\Omega) .
$$

Remark 4.1. The operator $A$ defined by (22) is linear, bounded, symmetric and compact due to compact embedding $W^{1,2} \hookrightarrow \hookrightarrow L^{2}$. Simple calculation gives that the eigenvalues of the operator $A$ are $\mu_{j}=\frac{1}{\kappa_{j}}, j=1,2, \ldots$ for $\Gamma_{D} \neq \emptyset$ and $\mu_{j}=\frac{1}{\kappa_{j}+1}, j=0,1,2, \ldots$ for $\Gamma_{D}=\emptyset$, and the corresponding eigenvectors of $A$ coincide with the eigenfunctions $e_{j}$ of (16). In particular, the maximal eigenvalue of $A$ is always one and therefore $(A u, u) \leq\|u\|_{H_{D}^{1}}^{2}$, where the equality holds only for all multiples $u$ of $e_{1}$ or $e_{0}$ if $\Gamma_{D} \neq \emptyset$ or $\Gamma_{D}=\emptyset$, respectively, see also Remark 2.1. Hence, $((I-A) u, u)>0$ for all $u \notin \operatorname{span}\left\{e_{1}\right\}$ in the case $\Gamma_{D} \neq \emptyset$ and for all $u \notin \operatorname{span}\left\{e_{0}\right\}$ in the case $\Gamma_{D}=\emptyset$.

We define two non-linear operators $N_{1}, N_{2}: H_{D}^{1} \times H_{D}^{1} \mapsto H_{D}^{1}$ as

$$
\left(N_{i}(u, v), \varphi\right)=\int_{\Omega} n_{i}(u, v) \varphi d \Omega \quad \text { for all } u, v, \varphi \in H_{D}^{1}, i=1,2 .
$$

These two operators are well-defined and continuous due to the theorem about Nemytskii operators and the assumptions (9).

Remark 4.2. It is known that under the assumptions (6),(9) we have

$$
\lim _{\|u\|_{H_{D}^{1}}+\|v\|_{H_{D}^{1}} \rightarrow 0} \frac{N_{i}(u, v)}{\|u\|_{H_{D}^{1}}+\|v\|_{H_{D}^{1}}}=0, \quad i=1,2 .
$$

For details see e.g. Appendix A.1 of [5].

Furthermore we define operators $\beta^{-}, \beta^{+}: H_{D}^{1} \mapsto H_{D}^{1}$ by

$$
\left(\beta^{\mp}(u), \varphi\right)=\mp \int_{\Omega} s_{\mp} u^{\mp} \varphi d \Omega \quad \text { for all } u, \varphi \in H_{D}^{1}
$$

and $\beta: H_{D}^{1} \mapsto H_{D}^{1}$ as

$$
\beta:=\beta^{+}+\beta^{-} .
$$

Due to the theorem about Nemytskii operators and (10) we can also define operators $\tilde{F}_{-}, \tilde{F}_{+}$: $H_{D}^{1} \mapsto H_{D}^{1}$ by

$$
\left(\tilde{F}_{\mp}(u), \varphi\right)=\mp \int_{\Omega} \tilde{f}_{\mp}\left(\mathbf{x}, u^{\mp}\right) \varphi d \Omega \quad \text { for all } u, \varphi \in H_{D}^{1}
$$

and $\tilde{F}: H_{D}^{1} \mapsto H_{D}^{1}$ as

$$
\tilde{F}:=\tilde{F}_{+}+\tilde{F}_{-} .
$$

Lemma 4.1. The operator $\beta$ is positively homogeneous (i.e. $\beta(t u)=t \beta(u)$ for all $t>0, u \in H_{D}^{1}$ ) and

$$
\begin{aligned}
& \text { i) } \exists c \in \mathbb{R}:\|\beta(u)\|_{H_{D}^{1}} \leq c\left\|s_{-}\right\|_{\infty}\left\|u^{-}\right\|_{H_{D}^{1}}+c\left\|s_{+}\right\|_{\infty}\left\|u^{+}\right\|_{H_{D}^{1}} \quad \forall u \in H_{D}^{1}, \\
& \text { ii) } u_{n} \rightarrow u \Longrightarrow \beta\left(u_{n}\right) \rightarrow \beta(u), \\
& \text { iii) }(\beta(u), u) \geq 0 \quad \forall u \in H_{D}^{1}, \\
& \text { iv) } u_{n} \rightarrow 0, \frac{u_{n}}{\left\|u_{n}\right\|_{H_{D}^{1}}} \rightarrow w \Longrightarrow \frac{\tilde{F}\left(u_{n}\right)}{\left\|u_{n}\right\|_{H_{D}^{1}}} \rightarrow \beta(w) .
\end{aligned}
$$


Proof.

The positive homogeneity is apparent.

i) Using the continuous embedding $H_{D}^{1} \hookrightarrow L^{2}$ and Hölder's inequality we get

$$
\begin{aligned}
\|\beta(u)\| & =\sup _{\|\varphi\|_{H_{D}^{1}} \leq 1}|(\beta(u), \varphi)|=\sup _{\|\varphi\|_{H_{D}^{1}} \leq 1}\left|\int_{\Omega} s_{+} u^{+} \varphi d \Omega-\int_{\Omega} s_{-} u^{-} \varphi d \Omega\right| \leq \\
& \leq\left\|s_{+}\right\|_{\infty} \sup _{\|\varphi\|_{H_{D}^{1}} \leq 1}\left\{\left\|u^{+}\right\|_{L^{2}} \cdot\|\varphi\|_{L^{2}}\right\}+\left\|s_{-}\right\|_{\infty} \sup _{\|\varphi\|_{H_{D}^{1}} \leq 1}\left\{\left\|u^{-}\right\|_{L^{2}} \cdot\|\varphi\|_{L^{2}}\right\} \leq \\
& \leq c\left\|s_{+}\right\|_{\infty} \sup _{\|\varphi\|_{H_{D}^{1} \leq 1}}\left\{\left\|u^{+}\right\|_{H_{D}^{1}} \cdot\|\varphi\|_{H_{D}^{1}}\right\}+c\left\|s_{-}\right\|_{\infty} \sup _{\|\varphi\|_{H_{D}^{1}} \leq 1}\left\{\left\|u^{-}\right\|_{H_{D}^{1}} \cdot\|\varphi\|_{H_{D}^{1}}\right\} \leq \\
& \leq c\left\|s_{+}\right\|_{\infty}\left\|u^{+}\right\|_{H_{D}^{1}}+c\left\|s_{-}\right\|_{\infty}\left\|u^{-}\right\|_{H_{D}^{1}} .
\end{aligned}
$$

ii) Let's have a sequence $\left(u_{n}\right) \subset H_{D}^{1}$ such that $u_{n} \rightarrow u \in H_{D}^{1}$. Then by the compact embedding $W^{1,2} \hookrightarrow \hookrightarrow L^{2}$, we get $u_{n} \rightarrow u$ in $L^{2}$. It is easy to see that $\left|u_{n}^{-}-u^{-}\right| \leq \mid u_{n}-$ $u \mid$ holds almost everywhere on $\Omega$. Hence,

$$
\begin{aligned}
\left\|\beta^{-}\left(u_{n}\right)-\beta^{-}(u)\right\|_{H_{D}^{1}} & =\sup _{\|\varphi\|_{H_{D}^{1}} \leq 1}\left|\left(\beta^{-}\left(u_{n}\right)-\beta^{-}(u), \varphi\right)\right| \leq \sup _{\|\varphi\|_{H_{D}^{1}} \leq 1} \int_{\Omega}\left|u_{n}^{-}-u^{-}\right| \cdot|\varphi| d \Omega \leq \\
& \leq C\left\|u_{n}-u\right\|_{L^{2}} \rightarrow 0 .
\end{aligned}
$$

The same can be shown for $\beta^{+}$and the assertion follows.

iii) Let $u \in H_{D}^{1}$ be arbitrary and $\Omega_{+}, \Omega_{-}$subsets of the domain $\Omega$ such that $\Omega=\Omega_{+} \cup \Omega_{-}, u \geq 0$ a.e. on $\Omega_{+}$and $u<0$ a.e. on $\Omega_{-}$. Hence

$$
(\beta(u), u)=\int_{\Omega} s_{+} u^{+} u d \Omega-\int_{\Omega} s_{-} u^{-} u d \Omega=\int_{\Omega_{+}} s_{+} u^{2} d \Omega_{+}+\int_{\Omega_{-}} s_{-} u^{2} d \Omega_{-}
$$

and our assertion follows.

iv) Now we will define a new auxiliary operator $F: H_{D}^{1} \mapsto H_{D}^{1}$ by

$$
(F(u), \varphi)=-\int_{\Omega}\left(\tilde{f}_{-}(\mathbf{x}, u)-s_{-} u\right) \varphi d \Omega \quad \text { for all } u, \varphi \in H_{D}^{1}
$$

We have

$$
\lim _{\xi \rightarrow 0} \frac{\tilde{f}_{-}(\mathbf{x}, \xi)-s_{-} \xi}{\xi}=0 \quad \text { for a.a. } \mathbf{x} \in \Omega
$$

by assumption (2). The growth conditions (10) and Proposition 3.2 of [4] give

$$
\lim _{u \rightarrow 0} \frac{F(u)}{\|u\|_{H_{D}^{1}}}=0 .
$$

If $u_{n} \rightarrow 0$, then $u_{n}^{-} \rightarrow 0$ (see [13]) and using (33) we get

$$
\lim _{n \rightarrow+\infty} \frac{\left\|\tilde{F}_{-}\left(u_{n}\right)-\beta^{-}\left(u_{n}\right)\right\|_{H_{D}^{1}}}{\left\|u_{n}\right\|_{H_{D}^{1}}}=\lim _{n \rightarrow+\infty} \frac{\left\|F\left(u_{n}^{-}\right)\right\|_{H_{D}^{1}}}{\left\|u_{n}\right\|_{H_{D}^{1}}} \leq \lim _{n \rightarrow+\infty} \frac{\left\|F\left(u_{n}^{-}\right)\right\|_{H_{D}^{1}}}{\left\|u_{n}^{-}\right\|_{H_{D}^{1}}}=0 .
$$

If $u_{n} \rightarrow 0, \frac{u_{n}}{\left\|u_{n}\right\|_{H_{D}^{1}}} \rightarrow w$ then

$$
\frac{\tilde{F}_{-}\left(u_{n}\right)}{\left\|u_{n}\right\|_{H_{D}^{1}}} \rightarrow \beta^{-}(w)
$$

due to positive homogeneity of $\beta^{-}$and (30).

The same can be shown for $\tilde{F}_{+}$and $\beta^{+}$and the assertion is proved. 
In order to give an operator formulation of the problem (11) or (12) with unilateral sources and sinks on the boundary (20), we define operators $\beta_{N}^{ \pm}: H_{D}^{1} \mapsto H_{D}^{1}$ as

$$
\left(\beta_{N}^{\mp}(u), \varphi\right)=\mp \int_{\Gamma_{N}} s_{\mp} u^{\mp} \varphi d \Gamma_{N} \quad \text { for all } u, \varphi \in H_{D}^{1}
$$

and $\beta_{N}: H_{D}^{1} \mapsto H_{D}^{1}$ as

$$
\beta_{N}=\beta_{N}^{+}+\beta_{N}^{-} .
$$

Remark 4.3. The operator $\beta_{N}$ possess the same properties as the operator $\beta$ (see Lemma 4.1).

Let's emphasize that for cases $\Gamma_{D}=\emptyset$ and $\Gamma_{D} \neq \emptyset$ we have two different inner products and therefore operators defined above are in these two cases also different. In the case $\Gamma_{D} \neq \emptyset$ we consider the function space $H_{D}^{1}$ equipped with the inner product $(u, \varphi)=\int_{\Omega} \nabla u \nabla \varphi d \Omega$. A weak solution of the problem (8),(4) or (5),(4) is then a pair of functions $u, v \in H_{D}^{1}$ satisfying

$$
\begin{aligned}
d_{1} u-b_{1,1} A u-b_{1,2} A v+\beta(u) & =0, \\
d_{2} v-b_{2,1} A u-b_{2,2} A v & =0
\end{aligned}
$$

or

$$
\begin{aligned}
d_{1} u-b_{1,1} A u-b_{1,2} A v-N_{1}(u, v)+\tilde{F}(u) & =0, \\
d_{2} v-b_{2,1} A u-b_{2,2} A v-N_{2}(u, v) & =0,
\end{aligned}
$$

respectively.

If $\Gamma_{D}=\emptyset$, the function space $H_{D}^{1}$ is identical with $W^{1,2}$ and is equipped with the inner product $(u, \varphi)=\int_{\Omega}(\nabla u \nabla \varphi+u \varphi) d \Omega$. A weak solution of (8),(4) or (5),(4) is then a pair of functions $u, v \in W^{1,2}$ satisfying

$$
\begin{aligned}
d_{1}(I-A) u-b_{1,1} A u-b_{1,2} A v+\beta(u) & =0, \\
d_{2}(I-A) v-b_{2,1} A u-b_{2,2} A v & =0
\end{aligned}
$$

or

$$
\begin{aligned}
d_{1}(I-A) u-b_{1,1} A u-b_{1,2} A v-N_{1}(u, v)+\tilde{F}(u) & =0 \\
d_{2}(I-A) v-b_{2,1} A u-b_{2,2} A v-N_{2}(u, v) & =0
\end{aligned}
$$

respectively.

For the problem (11), (20) or (12), (20) we will get analogous systems, we just replace operators $\beta$ and $\tilde{F}$ with $\beta_{N}$.

\section{Critical points for fixed $d_{2}$}

In this Section we will assume that $d_{2}>0$ is fixed and we will use the notation from Sections 2 and 4. As usually, by an eigenvalue of a positively homogeneous operator $P$ we mean a number $\lambda$ such that the equation $P(u)=\lambda u$ has a non-trivial solution. More generally, by an eigenvalue of a problem with a positively homogeneous operator we mean a parameter for which the problem under consideration has a non-trivial solution.

\subsection{Reduction to one operator equation for the case $\Gamma_{D} \neq \emptyset$}

Let's suppose $\Gamma_{D} \neq \emptyset$. Since the operator $A$ is positive by Remark 4.1 and $b_{2,2}<0$ by the assumption (7), the number $\frac{d_{2}}{b_{2,2}}$ is not its eigenvalue. Therefore the operator $d_{2} I-b_{2,2} A$ is invertible and surjective. Hence, we can express $v$ from the second equation in (36), substitute it into the first one and get

$$
d_{1} u-b_{1,1} A u-b_{1,2} A\left(d_{2} I-b_{2,2} A\right)^{-1} b_{2,1} A u+\beta(u)=0 .
$$


Introducing the operator $S_{d_{2}}: H_{D}^{1} \mapsto H_{D}^{1}$ as

$$
S_{d_{2}}:=b_{1,1} A+b_{1,2} A\left(d_{2} I-b_{2,2} A\right)^{-1} b_{2,1} A,
$$

we can write the system (36) as

$$
\begin{array}{r}
d_{1} u-S_{d_{2}} u+\beta(u)=0, \\
v=\left(d_{2} I-b_{2,2} A\right)^{-1} b_{2,1} A u .
\end{array}
$$

In particular, the system of the operator equations

$$
\begin{array}{r}
d_{1} u-S_{d_{2}} u=0, \\
v=\left(d_{2} I-b_{2,2} A\right)^{-1} b_{2,1} A u
\end{array}
$$

is equivalent with the system

$$
\begin{aligned}
& d_{1} u-b_{1,1} A u-b_{1,2} A v=0, \\
& d_{2} v-b_{2,1} A u-b_{2,2} A v=0 .
\end{aligned}
$$

Remark 5.1. The operator $S_{d_{2}}: H_{D}^{1} \mapsto H_{D}^{1}$ defined by (40) is linear, bounded, symmetric and compact. It follows from simple calculations and Remark 4.1 that the eigenvalues of the operator $S_{d_{2}}$ are

$$
d_{1}^{j}=\frac{1}{\kappa_{j}}\left(\frac{b_{1,2} b_{2,1}}{d_{2} \kappa_{j}-b_{2,2}}+b_{1,1}\right), j=1,2, \ldots
$$

and since $\kappa_{j} \rightarrow \infty$ as $j \rightarrow \infty$, we get $d_{1}^{j} \rightarrow 0$ as $j \rightarrow \infty$. The eigenvectors of $S_{d_{2}}$ corresponding to $d_{1}^{j}$ coincide with those of the operator A corresponding to $\mu_{j}$, i.e. with the eigenfunctions of (16) corresponding to $\kappa_{j}$.

\subsection{Reduction to one operator equation for the case $\Gamma_{D}=\emptyset$}

Let's consider the case $\Gamma_{D}=\emptyset$. It follows from Remark 4.1 that the number $d_{2}$ is not an eigenvalue of the operator $d_{2} A+b_{2,2} A$. Indeed, we have $d_{2} \neq \frac{d_{2}+b_{2,2}}{\kappa_{j}+1}$, because $d_{2} \kappa_{j} \neq b_{2,2}\left(b_{2,2}\right.$ is negative by (7)). Hence, the operator $d_{2} I-d_{2} A-b_{2,2} A$ (in (38)) is surjective and invertible. Similarly as in Section 5.1 we can transform the system (38) to the system

$$
\begin{gathered}
d_{1}(I-A) u-S_{d_{2}} u+\beta(u)=0, \\
v=\left(d_{2} I-d_{2} A-b_{2,2} A\right)^{-1} b_{2,1} A u,
\end{gathered}
$$

with the new operator

$$
S_{d_{2}}:=b_{1,1} A+b_{1,2} A\left(d_{2} I-d_{2} A-b_{2,2} A\right)^{-1} b_{2,1} A .
$$

In particular, the system of the operator equations

$$
\begin{array}{r}
d_{1}(I-A) u-S_{d_{2}} u=0, \\
v=\left(d_{2} I-d_{2} A-b_{2,2} A\right)^{-1} b_{2,1} A u
\end{array}
$$

is equivalent with the system

$$
\begin{aligned}
& d_{1}(I-A) u-b_{1,1} A u-b_{1,2} A v=0, \\
& d_{2}(I-A) v-b_{2,1} A u-b_{2,2} A v=0 .
\end{aligned}
$$

Remark 5.2. The operator $S_{d_{2}}$ defined by (46) is linear, continuous, symmetric and compact. Simple calculations and Remark 4.1 imply that the eigenvalues of the operator $S_{d_{2}}$ are

$$
\lambda^{j}=\frac{1}{\kappa_{j}+1}\left(\frac{b_{1,2} b_{2,1}}{d_{2} \kappa_{j}-b_{2,2}}+b_{1,1}\right), j=0,1,2, \ldots
$$

and the eigenvectors of $S_{d_{2}}$ corresponding to $\lambda^{j}$ coincide with those of $A$ corresponding to $\mu_{j}$, i.e. with the eigenfunctions of (16) corresponding to $\kappa_{j}$. However, the eigenvalues $d_{1}^{j}$ of the problem (47a) are the same as those of the operator $S_{d_{2}}$ defined by (40) in the case $\Gamma_{D} \neq \emptyset$, i.e. they are given by (44). (There is no eigenvalue with $j=0$.) 


\subsection{Maximal eigenvalues and critical points}

Notation 5.1. We will denote by $d_{1}^{M A X}$ the maximal eigenvalue of the operator $S_{d_{2}}$ or of the problem $(47 a)$ in the case $\Gamma_{D} \neq \emptyset$ or $\Gamma_{D}=\emptyset$, respectively. We will also denote by $d_{1}^{M A X, \beta}$ the maximal eigenvalue of the operator $S_{d_{2}}-\beta$ or of the problem (45a) in the case $\Gamma_{D} \neq \emptyset$ or $\Gamma_{D}=\emptyset$, respectively, if it exists.

Observation 5.1. We can see from the form (44) of the eigenvalues $d_{1}^{j}$ (see Remarks 5.1, 5.2) and from (17), that a point $\left[d_{1}, d_{2}\right]$ lies on a hyperbola $C_{j}$ for some $j \in \mathbb{N}$ if and only if $d_{1}$ is an eigenvalue of $S_{d_{2}}$ in the case $\Gamma_{D} \neq \emptyset$ or an eigenvalue of (47a) in the case $\Gamma_{D}=\emptyset$. For the maximal eigenvalue $d_{1}^{M A X}$ of $S_{d_{2}}$ in the case $\Gamma_{D} \neq \emptyset$ and of (47a) in the case $\Gamma_{D}=\emptyset$ we have $\left[d_{1}^{M A X}, d_{2}\right] \in C_{E}$. It follows from (44) and Remark 5.2 that the operator $S_{d_{2}}$ in the case $\Gamma_{D} \neq \emptyset$ and the problem (47a) in the case $\Gamma_{D}=\emptyset$ have infinitely many positive eigenvalues and maximally finite number of negative eigenvalues. See also Figure 1.

Lemma 5.1. If $\Gamma_{D} \neq \emptyset$, then a point $\left[d_{1}, d_{2}\right] \in \mathbb{R}_{+}^{2}$ is a critical point of the system (11),(4) or (8), (4) if and only if $d_{1}$ is an eigenvalue of the operator $S_{d_{2}}$ or $S_{d_{2}}-\beta$, respectively.

If $\Gamma_{D}=\emptyset$, then a point $\left[d_{1}, d_{2}\right] \in \mathbb{R}_{+}^{2}$ is a critical point of the system (11), (4) or (8), (4) if and only if $d_{1}$ is an eigenvalue of the problem (47a) or (45a), respectively.

Proof. Let $\Gamma_{D} \neq \emptyset$. A point $\left[d_{1}, d_{2}\right] \in \mathbb{R}_{+}^{2}$ is a critical point of the system (11), (4) or (8), (4) if and only if there exists a non-trivial solution $[u, v]$ of (43) or (36), respectively. This is true if and only if there exists a non-trivial solution $u \in H_{D}^{1}$ of $(42 a)$ or $(41 a)$, i.e. $d_{1}$ is an eigenvalue of the operator $S_{d_{2}}$ or $S_{d_{2}}-\beta$, respectively (see Section 5.1). The proof for the case $\Gamma_{D}=\emptyset$ is analogous, we only use (48), (38), (47a), (45a) and the result of Section 5.2.

We will use a variational characterization of the largest eigenvalue of an eigenvalue problem with a positively homogeneous operator to a study of critical points of the problem (8), (4). The following abstract theorem is a slight modification of the result proved for the particular case $L \equiv 0$ in $[6]$ and for the general case in a forthcoming paper of J. Navrátil. Let's remind that $\operatorname{Ker}(I-L)$ is the kernel of the operator $I-L$.

Theorem 5.1. Let $H$ be a Hilbert space, $P: H \mapsto H$ a positively homogeneous, continuous operator such that

$$
u_{n} \rightarrow u \Longrightarrow P\left(u_{n}\right) \rightarrow P(u)
$$

and $L: H \mapsto H$ a linear, continuous, symmetric and compact operator. In the case $L \not \equiv 0$ we suppose that the maximal eigenvalue of $L$ is in the interval $(0,1]$. Let there exist $u_{0} \in H, u_{0} \notin$ $\operatorname{Ker}(I-L)$ such that

$$
\lambda_{0}:=\max _{\substack{u \in H \\ u \notin \operatorname{Ker}(I-L)}} \frac{(P(u), u)}{((I-L) u, u)}=\frac{\left(P\left(u_{0}\right), u_{0}\right)}{\left((I-L) u_{0}, u_{0}\right)}>0
$$

and

$$
\lim _{t \rightarrow 0} \frac{1}{t}\left(P\left(u_{0}+t h\right)-P\left(u_{0}\right), u_{0}\right)=\left(P\left(u_{0}\right), h\right) \quad \forall h \in H .
$$

Then $\lambda_{0}$ is the maximal eigenvalue of the problem

$$
\lambda(I-L) u-P(u)=0
$$

and $u_{0}$ is a corresponding eigenvector. If $u_{1} \notin \operatorname{Ker}(I-L)$ is an arbitrary eigenvector of (52) corresponding to $\lambda_{0}$ then it satisfies (50) with $u_{0}$ replaced by $u_{1}$.

Let us note that the problem (52) has an eigenvector in $\operatorname{Ker}(I-L)$ only if there is $u \in \operatorname{Ker}(I-$ $L$ ) such that $P(u)=0$. In this case any $\lambda$ is an eigenvalue. 
Proof.

We will assume that $L \not \equiv 0$, the case $L \equiv 0$ is simpler. Let us denote by $\mu_{L}^{M A X}$ the maximal eigenvalue of $L$. Since $\mu_{L}^{M A X} \in(0,1]$, we have $\max _{\substack{u \neq 0 \\\|u\|_{H_{D}^{1}}=1}}(L u, u)=\mu_{L}^{M A X} \leq 1$. If $\mu_{L}^{M A X}<1$, then $(L u, u)<1$ and therefore $((I-L) u, u)>0$ for all $u$. If $\mu_{L}^{M A X}=1$, then $\max _{\substack{u \neq 0 \\\|u\|_{H_{D}^{1}}=1}}(L u, u)=1$, but the maximum is attained only in the elements of $\operatorname{Ker}(I-A)$. Hence, $((I-L) u, u)>0$ for all $u \notin \operatorname{Ker}(I-L)$ and the expression in (50) makes sense.

Let $u_{0} \notin \operatorname{Ker}(I-L)$ be arbitrary such that (50) and (51) are fulfilled, and let $h \in H_{D}^{1}$ be arbitrary fixed. Then for $t \in \mathbb{R}$ small such that $\left(u_{0}+t h\right) \notin \operatorname{Ker}(I-L)$ we have

$$
\frac{\left(P\left(u_{0}+t h\right), u_{0}+t h\right)}{\left((I-L)\left(u_{0}+t h\right), u_{0}+t h\right)} \leq \frac{\left(P\left(u_{0}\right), u_{0}\right)}{\left((I-L) u_{0}, u_{0}\right)}=: \lambda_{0}
$$

We can rewrite this inequality as

$$
\left(P\left(u_{0}+t h\right), u_{0}\right)+t\left(P\left(u_{0}+t h\right), h\right) \leq \frac{\left(P\left(u_{0}\right), u_{0}\right)}{\left((I-L) u_{0}, u_{0}\right)}\left[\left((I-L) u_{0}, u_{0}\right)+2 t\left((I-L) u_{0}, h\right)+t^{2}((I-L) h, h)\right]
$$

and eventually as

$$
\left(P\left(u_{0}+t h\right), u_{0}\right)-\left(P\left(u_{0}\right), u_{0}\right)+t\left(P\left(u_{0}+t h\right), h\right) \leq \lambda_{0}\left[2 t\left((I-L) u_{0}, h\right)+t^{2}((I-L) h, h)\right] .
$$

We divide it by $2 t$ and get

$$
\begin{array}{ll}
\frac{1}{2 t}\left[\left(P\left(u_{0}+t h\right), u_{0}\right)-\left(P\left(u_{0}\right), u_{0}\right)\right]+\frac{1}{2}\left(P\left(u_{0}+t h\right), h\right) \leq \lambda_{0}\left[\left((I-L) u_{0}, h\right)+\frac{t}{2}((I-L) h, h)\right], \quad t>0, \\
\frac{1}{2 t}\left[\left(P\left(u_{0}+t h\right), u_{0}\right)-\left(P\left(u_{0}\right), u_{0}\right)\right]+\frac{1}{2}\left(P\left(u_{0}+t h\right), h\right) \geq \lambda_{0}\left[\left((I-L) u_{0}, h\right)+\frac{t}{2}((I-L) h, h)\right], \quad t<0 .
\end{array}
$$

Let $t \rightarrow 0$. We use the condition (51) and continuity of $P$ to get

$$
\begin{aligned}
& \left(P\left(u_{0}\right), h\right) \leq \lambda_{0}\left((I-L) u_{0}, h\right), \\
& \left(P\left(u_{0}\right), h\right) \geq \lambda_{0}\left((I-L) u_{0}, h\right) .
\end{aligned}
$$

Since $h$ was arbitrary, we have

$$
\left(P\left(u_{0}\right), h\right)=\lambda_{0}\left((I-L) u_{0}, h\right) \quad \text { for all } h \in H_{D}^{1},
$$

that means

$$
P\left(u_{0}\right)=\lambda_{0}(I-L) u_{0}
$$

Hence, the number $\lambda_{0}$ is an eigenvalue of the problem (52) and $u_{0}$ is a corresponding eigenvector.

Let $\lambda_{1}$ be another eigenvalue of the problem (52) and let $u_{1} \notin \operatorname{Ker}(I-L)$ be a corresponding eigenvector. Then we have

$$
P\left(u_{1}\right)=\lambda_{1}(I-L) u_{1}
$$

and if we multiply it by $u_{1}$ and divide by $\left((I-L) u_{1}, u_{1}\right)$, we get

$$
\lambda_{1}=\frac{\left(P\left(u_{1}\right), u_{1}\right)}{\left((I-L) u_{1}, u_{1}\right)} \leq \frac{\left(P\left(u_{0}\right), u_{0}\right)}{\left((I-L) u_{0}, u_{0}\right)}=\lambda_{0} .
$$

Hence, $\lambda_{0}$ is the maximal eigenvalue. If $\lambda_{1}=\lambda_{0}$, then we have equality in the last estimate, that means $u_{1}$ is a maximizer of the expression (50). That means an arbitrary eigenvector corresponding to $\lambda_{0}$ not lying in $\operatorname{Ker}(I-L)$ satisfies (50) with $u_{0}$ replaced by $u_{1}$.

If the condition (51) is fulfilled for any $u_{0}$, then it actually means that $P$ has a potential $\Phi=$ $\frac{1}{2}(P u, u)$. 
Remark 5.3. In the particular case $L \equiv 0, \lambda_{0}:=\max _{\substack{u \in H \\ u \neq 0}} \frac{(P(u), u)}{\|u\|_{H}^{2}}$ is the maximal eigenvalue of $P$.

Theorem 5.2. Let $\Gamma_{D} \neq \emptyset$ and let $S_{d_{2}}$ be the operator from (40). If there exists a function $\varphi \in H_{D}^{1}$ such that

$$
\left(S_{d_{2}} \varphi, \varphi\right)-(\beta(\varphi), \varphi)>0,
$$

then the maximal eigenvalue of the operator $S_{d_{2}}-\beta$ is

$$
d_{1}^{M A X, \beta}:=\max _{\substack{u \in H_{D}^{1} \\ u \neq o}} \frac{\left(S_{d_{2}} u, u\right)-(\beta(u), u)}{\|u\|_{H_{D}^{1}}^{2}}=\max _{\substack{u \in H_{D}^{1} \\\|u\|_{H_{D}^{1}}^{1}}}\left(S_{d_{2}} u, u\right)-(\beta(u), u)>0 .
$$

Maximizers of the expression in (54) are exactly all eigenvectors of $S_{d_{2}}-\beta$ corresponding to $d_{1}^{M A X, \beta}$.

Proof.

Let's prove that the maximum in (54) exists. Let

$$
M:=\sup _{\substack{u \in H_{D}^{1} \\\|u\|_{H_{D}^{1}}^{1}=1}}\left(S_{d_{2}} u, u\right)-(\beta(u), u) .
$$

The existence of $\varphi$ satisfying (53) implies $M>0$. We can choose a sequence $\left(u_{n}\right) \subset H_{D}^{1}$ with $\left\|u_{n}\right\|_{H_{D}^{1}}=1$ such that

$$
\lim _{n \rightarrow \infty}\left(S_{d_{2}} u_{n}, u_{n}\right)-\left(\beta\left(u_{n}\right), u_{n}\right)=M .
$$

We can assume $u_{n} \rightarrow u_{0} \in H_{D}^{1}$. Since $S_{d_{2}}$ is linear and compact and $\beta$ satisfies (30), we get

$$
\left(S_{d_{2}} u_{n}, u_{n}\right)-\left(\beta\left(u_{n}\right), u_{n}\right) \rightarrow\left(S_{d_{2}} u_{0}, u_{0}\right)-\left(\beta\left(u_{0}\right), u_{0}\right)=M .
$$

Now we will show that $\left\|u_{0}\right\|_{H_{D}^{1}}=1$. We know that $\left\|u_{0}\right\|_{H_{D}^{1}} \leq 1$. If $0<\left\|u_{0}\right\|_{H_{D}^{1}}<1$, then $\left(S_{d_{2}} \frac{u_{0}}{\left\|u_{0}\right\|_{H_{D}^{1}}}, \frac{u_{0}}{\left\|u_{0}\right\|_{H_{D}^{1}}}\right)-\left(\beta\left(\frac{u_{0}}{\left\|u_{0}\right\|_{H_{D}^{1}}}\right), \frac{u_{0}}{\left\|u_{0}\right\|_{H_{D}^{1}}}\right)=\frac{M}{\left\|u_{0}\right\|_{H_{D}^{1}}^{2}}>M$ due to positive homogeneity of $\beta$ (see Lemma 4.1), which contradicts the fact that $M$ is supremum. If $u_{0}=0$, then $M=0$, which is not the case. Therefore the last maximum in (54) exists and it is attained at $u_{0}$ with $\left\|u_{0}\right\|_{H_{D}^{1}}=1$. The equality between two maxima in (54) follows from the positive homogeneity of $\beta$.

It is known that $P=\beta$ and therefore also $P=S_{d_{2}}-\beta$ satisfies (51) for any $u_{0}$ (see Lemma $A .3$ in Appendix). The operator $P=S_{d_{2}}-\beta$ satisfies also the other assumptions of Theorem 5.1 (see Remark 5.1 and Lemma 4.1). Hence, the assertions of Theorem 5.2 follow from Theorem 5.1, where we choose $L=0$, that means we have $\operatorname{Ker}(I-L)=\{0\}$.

Remark 5.4. Let's consider the case $\Gamma_{D}=\emptyset$. The definition of the inner product and of the operator $A$ (Section 4) give $((I-A) u, \varphi)=\int_{\Omega}(\nabla u, \nabla \varphi) d \Omega$ for all $u, \varphi$. It follows that $(I-A) u=0$ is equivalent to $((I-A) u, u)=0$, and this holds if and only if $u$ is a constant function. In other words, $\operatorname{Ker}(I-A)=\operatorname{span}\left\{e_{0}\right\}, e_{0}$ being the eigenfunction of (16) corresponding to $\kappa_{0}$. Due to Remark 5.2, any non-trivial $u_{0} \in \operatorname{Ker}(I-A)$ is simultaneously an eigenvector of $S_{d_{2}}$ from (46) corresponding to $\lambda^{0}$. Hence, by using (7) we get

$$
\left(S_{d_{2}} u_{0}, u_{0}\right)=\left(\lambda^{0} u_{0}, u_{0}\right)=\left(b_{1,1}+\frac{b_{1,2} b_{2,1}}{-b_{2,2}}\right)\left\|u_{0}\right\|_{W^{1,2}}^{2}=\frac{-\operatorname{det}(\mathbf{B})}{-b_{2,2}}\left\|u_{0}\right\|_{W^{1,2}}^{2}<0 .
$$

Theorem 5.3. Let $\Gamma_{D}=\emptyset$ and let $S_{d_{2}}$ be the operator from (46). If there exists a function $\varphi \in W^{1,2}$ satisfying (53), then the maximal eigenvalue of the problem (45a) is

$$
d_{1}^{M A X, \beta}:=\max _{\substack{u \in W^{1,2} \\ u \notin K \operatorname{Ker}(I-A)}} \frac{\left(S_{d_{2}} u, u\right)-(\beta(u), u)}{((I-A) u, u)}>0 .
$$


Maximizers of the expression in (58) are exactly all eigenvectors of the problem (45a) corresponding to $d_{1}^{M A X, \beta}$.

Proof.

Let's denote

$$
M:=\sup _{\substack{u \in W^{1,2} \\ u \notin \operatorname{Ker}(I-A)}} \frac{\left(S_{d_{2}} u, u\right)-(\beta(u), u)}{((I-A) u, u)} .
$$

Since $((I-A) u, u)=\int_{\Omega}(\nabla u)^{2} d \Omega \geq 0$ for every $u$ and we assume that there exists a function $\varphi$ satisfying (53), we have $M>0$.

We can choose a sequence $u_{n} \notin \operatorname{Ker}(I-A)$ with $\left\|u_{n}\right\|_{W^{1,2}}=1$ such that

$$
\lim _{n \rightarrow \infty} \frac{\left(S_{d_{2}} u_{n}, u_{n}\right)-\left(\beta\left(u_{n}\right), u_{n}\right)}{\left((I-A) u_{n}, u_{n}\right)}=M .
$$

We can assume that $u_{n} \rightarrow u_{0}$. If $u_{0}=0$, then we have

$$
\left((I-A) u_{n}, u_{n}\right)=1-\left(A u_{n}, u_{n}\right) \rightarrow 1-\left(A u_{0}, u_{0}\right)=1
$$

due to the compactness of $A$, and

$$
\left(S_{d_{2}} u_{n}, u_{n}\right)-\left(\beta\left(u_{n}\right), u_{n}\right) \rightarrow\left(S_{d_{2}} u_{0}, u_{0}\right)-\left(\beta\left(u_{0}\right), u_{0}\right)=0
$$

by the compactness of $S_{d_{2}}$ and (30). This means that $M=0$, which contradicts the positivity of $M$.

Further, let's show that $u_{0} \notin \operatorname{Ker}(I-A) \backslash\{0\}$, i.e. $u_{0}$ is not a constant function. Let $u_{0}$ be a non-zero constant function. Then $\left(S_{d_{2}} u_{0}, u_{0}\right)<0$ by Remark 5.4. Since we have $-(\beta(u), u) \leq 0$ for every $u$ by $(31)$, we get $\left(S_{d_{2}} u_{0}, u_{0}\right)-\left(\beta\left(u_{0}\right), u_{0}\right)<0$ and consequently

$$
\lim _{n \rightarrow \infty} \frac{\left(S_{d_{2}} u_{n}, u_{n}\right)-\left(\beta\left(u_{n}\right), u_{n}\right)}{\left((I-A) u_{n}, u_{n}\right)} \leq 0 .
$$

That contradicts the fact that $u_{n}$ is a maximizing sequence and the supremum $M$ is positive. Hence, we have $u_{0} \notin \operatorname{Ker}(I-L)$.

We need to show that $\left\|u_{0}\right\|_{W^{1,2}}=1$. We already know that $0<\left\|u_{0}\right\|_{W^{1,2}} \leq 1$. Now let $0<\left\|u_{0}\right\|_{W^{1,2}}<1$. We have $1-\left(A u_{0}, u_{0}\right)>0$ (see Remark 4.1) and

$$
\frac{\left(S_{d_{2}} u_{n}, u_{n}\right)-\left(\beta\left(u_{n}\right), u_{n}\right)}{\left((I-A) u_{n}, u_{n}\right)} \rightarrow \frac{\left(S_{d_{2}} u_{0}, u_{0}\right)-\left(\beta\left(u_{0}\right), u_{0}\right)}{1-\left(A u_{0}, u_{0}\right)}=M
$$

by the compactness of $S_{d_{2}}, A$ and the condition (30). Simultaneously $\left\|u_{0}\right\|_{W^{1,2}}^{2}-\left(A u_{0}, u_{0}\right)>0$ because of $u_{0} \notin \operatorname{Ker}(I-A)$ (see Remarks 4.1 and 5.4). It follows that

$$
\frac{\left(S_{d_{2}} u_{0}, u_{0}\right)-\left(\beta\left(u_{0}\right), u_{0}\right)}{\left\|u_{0}\right\|_{W^{1,2}}^{2}-\left(A u_{0}, u_{0}\right)}>\frac{\left(S_{d_{2}} u_{0}, u_{0}\right)-\left(\beta\left(u_{0}\right), u_{0}\right)}{1-\left(A u_{0}, u_{0}\right)}=M>0,
$$

which contradicts that fact that $M$ is a supremum. Hence, we have $\left\|u_{0}\right\|_{W^{1,2}}=1$.

We use compactness of $S_{d_{2}}, A$, the property (30) of $\beta$ and the fact that $\left\|u_{n}\right\|_{W^{1,2}}=1=$ $\left\|u_{0}\right\|_{W^{1,2}}$ to get

$$
\frac{\left(S_{d_{2}} u_{n}, u_{n}\right)-\left(\beta\left(u_{n}\right), u_{n}\right)}{\left((I-A) u_{n}, u_{n}\right)} \rightarrow \frac{\left(S_{d_{2}} u_{0}, u_{0}\right)-\left(\beta\left(u_{0}\right), u_{0}\right)}{\left((I-A) u_{0}, u_{0}\right)} .
$$

Hence, the maximum exists and it is attained at the function $u_{0} \notin \operatorname{Ker}(I-A)$ with $\left\|u_{0}\right\|_{W^{1,2}}=1$.

It is know that $P=S_{d_{2}}-\beta$ satisfies (51) for any $u_{0}$ (see Lemma $A .3$ in Appendix). The operators $P=S_{d_{2}}-\beta$ and $L=A$ also satisfy the other assumptions of Theorem 5.1 (see Remark 4.1, Remark 5.2 and Lemma 4.1). Hence, $d_{1}^{M A X, \beta}$ is the maximal eigenvalue and $u_{0}$ is a corresponding eigenvector of the problem (45a) by Theorem 5.1. 
Let's show that if $u_{1}$ is an arbitrary eigenvector of (45a) corresponding to $d_{1}^{M A X, \beta}$ then $u_{1} \notin \operatorname{Ker}(I-A)$. If $u_{1} \in \operatorname{Ker}(I-A) \backslash\{0\}$, then we have $-\left(S_{d_{2}} u_{1}, u_{1}\right)+\left(\beta\left(u_{1}\right), u_{1}\right)>0$ (see (57) and Lemma 4.1) and $d_{1}^{M A X, \beta}\left((I-A) u_{1}, u_{1}\right)=0$, which contradicts the equation (45a) with $u=u_{1}$ multiplied by $u_{1}$. Hence, $u_{1} \notin \operatorname{Ker}(I-A)$, and the last assertion of Theorem 5.3 follows also from Theorem 5.1.

Remark 5.5. The assumption (53) is clearly satisfied if there exists $\varphi$ such that $(\beta(\varphi), \varphi)=0$ and $\left(S_{d_{2}} \varphi, \varphi\right)>0$, which is easier to verify. If there is no $\varphi$ satisfying (53) then the supremum in proofs of Theorems 5.2 and 5.3 is not positive. It follows that there is no positive eigenvalue of the operator $S_{d_{2}}-\beta$ in the case $\Gamma_{D} \neq \emptyset$ or of the problem $(45 a)$ in the case $\Gamma_{D}=\emptyset$. Indeed:

- in the case $\Gamma_{D} \neq \emptyset$, if $d_{1}>0$ were an eigenvalue, then we would have $\left(S_{d_{2}} u, u\right)-(\beta(u), u)=$ $d_{1}(u, u)>0$ for the corresponding eigenvector $u$, which would contradict the non-positivity of the supremum.

- in the case $\Gamma_{D}=\emptyset$, an eigenvector $u$ cannot be constant (see the end of the proof of Theorem 5.3), and if $u$ were non-constant, then we would have $\left(S_{d_{2}} u, u\right)-(\beta(u), u)=d_{1}((I-A) u, u)>$ 0 by the last assertion of Remark 4.1, which would be a contradiction again.

It follows that in the situation of Theorem 3.4 there exists $\varphi$ satisfying (53) because that theorem guarantees the existence of a critical point in $D_{U} \cup C_{E}$ and consequently also existence of a positive eigenvalue of the operator $S_{d_{2}}-\beta$ in the case $\Gamma_{D} \neq \emptyset$ or of the problem (45a) in the case $\Gamma_{D}=\emptyset$ (see Sections 5.1, 5.2).

The following theorem is formulated for both cases $\Gamma_{D} \neq \emptyset$ and $\Gamma_{D}=\emptyset$.

Theorem 5.4. If $\left[d_{1}, d_{2}\right]$ is a critical point of (8), (4), then always $d_{1} \leq d_{1}^{M A X}$. If $\left[d_{1}^{M A X}, d_{2}\right] \in C_{i}$ exactly for $i=j, \ldots, j+k-1$, all linear combinations e of $e_{j}, \ldots, e_{j+k-1}$ satisfy (19) and $\left[d_{1}, d_{2}\right]$ is a critical point of (8),(4), then $d_{1}<d_{1}^{M A X}$. Moreover, if the assumption (53) is satisfied, then $d_{1} \leq d_{1}^{M A X, \beta}<d_{1}^{M A X}$.

The assumption concerning a position of $\left[d_{1}^{M A X}, d_{2}\right]$ is fulfilled either if $\left[d_{1}^{M A X}, d_{2}\right]$ lies in fact only on one hyperbola $C_{j}=\ldots C_{j+k-1}$ (the eigenvalue $\kappa_{j}$ has the multiplicity $k$ ) or in the intersection of two different hyperbolas $C_{j}=\ldots C_{j+l-1} \neq C_{j+l}=\ldots C_{j+k-1}\left(\kappa_{j}\right.$ has the multiplicity $l, \kappa_{j+l}$ has the multiplicity $k-l$ ). See also Remark 2.2. Cf. also comments after Theorem 3.1, where the assumptions are related to a set $C_{r}^{R}$, while in Theorem 5.4 they concern only one point $\left[d_{1}^{M A X}, d_{2}\right]$ with a given fixed $d_{2}$.

Proof.

First let's consider the case $\Gamma_{D} \neq \emptyset$.

Let's show that if (53) were fulfilled with no $\varphi$ then no critical point of (8), (4) with $d_{2}$ under consideration would exist. If $\left[d_{1}, d_{2}\right]$ were a critical point with $d_{1}>0$, then $d_{1}$ would be an eigenvalue of $S_{d_{2}}-\beta$ (see Lemma 5.1). Hence, we would have $u$ with $\|u\|_{H_{D}^{1}}=1$ satisfying (41a). It would follow that $\left(S_{d_{2}} u, u\right)-(\beta(u), u)=d_{1}\|u\|_{H_{D}^{1}}>0$ and the condition (53) would be satisfied with $\varphi=u$, which is a contradiction.

Hence, in the following we can assume that (53) is fulfilled with some $\varphi$. Due to Theorem 5.2 and Lemma 4.1 we get

$$
d_{1}^{M A X, \beta}=\max _{\substack{u \in H_{D}^{1} \\ u \neq 0}} \frac{\left(S_{d_{2}} u, u\right)-(\beta(u), u)}{\|u\|_{H_{D}^{1}}^{2}} \leq \max _{\substack{u \in H_{D}^{1} \\ u \neq 0}} \frac{\left(S_{d_{2}} u, u\right)}{\|u\|_{H_{D}^{1}}^{2}}=d_{1}^{M A X} .
$$

As above, if $\left[d_{1}, d_{2}\right]$ is a critical point (8), (4), then $d_{1}$ is an eigenvalue of $S_{d_{2}}-\beta$ (see Lemma 5.1). Hence, the first assertion of Theorem 5.4 is true.

There exists $u_{0} \in H_{D}^{1}$ such that

$$
d_{1}^{M A X, \beta}=\frac{\left(S_{d_{2}} u_{0}, u_{0}\right)-\left(\beta\left(u_{0}\right), u_{0}\right)}{\left\|u_{0}\right\|_{H_{D}^{1}}^{2}} .
$$


Due to Lemma 4.1 we have

$$
d_{1}^{M A X, \beta} \leq \frac{\left(S_{d_{2}} u_{0}, u_{0}\right)}{\left\|u_{0}\right\|_{H_{D}^{1}}^{2}} \leq d_{1}^{M A X}
$$

Let $\left(\beta\left(u_{0}\right), u_{0}\right)=0$. Let us show that then the last inequality is strict. Indeed, if we had equality in (60), then $u_{0}$ would be an eigenvector of $S_{d_{2}}$ corresponding to $d_{1}^{M A X}$, that means a linear combination of $e_{j}, \ldots, e_{j+k-1}$ (see Remark 5.1). Hence, (19) with $e$ replaced by $u$ would be fulfilled by our assumptions and we would get $\left(\beta\left(u_{0}\right), u_{0}\right)>0$. This contradiction implies that the inequality in (60) must be strict and we get $d_{1}^{M A X, \beta}<d_{1}^{M A X}$.

If $\left(\beta\left(u_{0}\right), u_{0}\right)>0$, then the first inequality in (60) is strict and consequently $d_{1}^{M A X, \beta}<d_{1}^{M A X}$ again.

If $\left[d_{1}, d_{2}\right]$ is a critical point of the problem $(8),(4)$, then $d_{1}$ is an eigenvalue of $S_{d_{2}}-\beta$ by Lemma 5.1 and therefore

$$
d_{1} \leq d_{1}^{M A X, \beta}<d_{1}^{M A X} .
$$

The proof for $\Gamma_{D}=\emptyset$ is analogous, but we must use Remark 5.2 and Theorem 5.3, in particular formula (58) instead of (54).

\section{Proofs of main results}

We will use notation from the previous sections.

\section{Proof of Theorem 3.1}

i) Since $d_{2}>0$ was arbitrary in Section 5 and $\left[d_{1}^{M A X}, d_{2}\right] \in C_{E}$ (see Observation 5.1 ), it follows from Theorem 5.4 that there are no critical points of (8), (4) in $D_{S}$ (see also Figure 1). Consequently there are also no bifurcation points of $(5),(4)$ in $D_{S}$ (see Lemma A.2 in Appendix).

ii) Let's consider the case $\Gamma_{D} \neq \emptyset$. Let's suppose the opposite, i.e. the assumptions of the second part of Theorem 3.1 are satisfied and there are critical points of $(8),(4)$ in $C_{r}^{R}(\varepsilon)$ for every $\varepsilon>0$. We can choose a sequence $d^{n}=\left[d_{1}^{n}, d_{2}^{n}\right] \in D_{U}$ and $W_{n}=\left[u_{n}, v_{n}\right]$ such that $d^{n} \rightarrow d^{0} \in C_{r}^{R}$, $\left\|W_{n}\right\|=\|u\|_{H_{D}^{1}}+\|v\|_{H_{D}^{1}} \neq 0$ and $d^{n}, W_{n}$ satisfy (36). We can assume that $\frac{W_{n}}{\left\|W_{n}\right\|} \rightarrow W=[w, z]$. Let's divide (36) by $\left\|W_{n}\right\|$ to get

$$
\begin{array}{r}
d_{1}^{n} \frac{u_{n}}{\left\|W_{n}\right\|}-b_{1,1} A \frac{u_{n}}{\left\|W_{n}\right\|}-b_{1,2} A \frac{v_{n}}{\left\|W_{n}\right\|}+\beta\left(\frac{u_{n}}{\left\|W_{n}\right\|}\right)=0, \\
d_{2}^{n} \frac{v_{n}}{\left\|W_{n}\right\|}-b_{2,1} A \frac{u_{n}}{\left\|W_{n}\right\|}-b_{2,2} A \frac{v_{n}}{\left\|W_{n}\right\|}=0 .
\end{array}
$$

By the compactness of $A$ and (30), we get $A \frac{u_{n}}{\left\|W_{n}\right\|} \rightarrow A w$ and $\beta\left(\frac{u_{n}}{\left\|W_{n}\right\|}\right) \rightarrow \beta(w)$, analogously for $v_{n}$ and $z$. Hence, it follows easily from (61) that $\frac{u_{n}}{\left\|W_{n}\right\|} \rightarrow w, \frac{v_{n}}{\left\|W_{n}\right\|} \rightarrow z$ and

$$
\begin{aligned}
d_{1}^{0} w-b_{1,1} A w-b_{1,2} A z-\beta(w) & =0 \\
d_{2}^{0} z-b_{2,1} A w-b_{2,2} A z & =0
\end{aligned}
$$

Therefore the point $d^{0}=\left[d_{1}^{0}, d_{2}^{0}\right] \in C_{r}^{R}$ is a critical point of the system $(8),(4)$, which contradicts Theorem 5.4 for $d_{2}=d_{2}^{0}$. Hence, there exists $\varepsilon>0$ such that there are no critical points of (8), (4) and consequently no bifurcation points of $(5),(4)$ in $C_{r}^{R}(\varepsilon)$ (see Lemma A.2 in Appendix).

The proof for $\Gamma_{D}=\emptyset$ is analogous, we only use the system (38) instead of the system (36). 


\section{Proof of Theorem 3.2}

i) Under the assumptions about $s_{ \pm}$, either $e=e_{1}$ or $e=-e_{1}$ satisfies $s_{-} e^{-}-s_{+} e^{+} \equiv 0$. Since any point $d \in C_{1}$ is a critical point of the problem (11),(4) with a non-trivial solution $\left[\frac{d_{2} \kappa_{1}-b_{2,2}}{b_{2,1}} e_{1}, e_{1}\right]$ due to Remark 2.3, it is also a critical point of the problem (8), (4).

ii) Due to the definition of $d_{2}^{I}$, for $d_{2}<d_{2}^{I}$ we have $\left[d_{1}^{M A X}, d_{2}\right] \in C_{j}$ for a finite number of indices $j>1$ (see Section 2 and also Observation 5.1). Any linear combination $e$ of the eigenfunctions $e_{j}, j>1$ changes the sign (see Lemma $A .1$ in Appendix). Hence, under the assumptions of Theorem 3.2 we have $s_{-} e^{-}-s_{+} e^{+} \not \equiv 0$. Therefore any critical point $\left[d_{1}, d_{2}\right]$ with $d_{2}<d_{2}^{I}$ of the problem (8), (4) satisfies $d_{1}<d_{1}^{M A X}$ by Theorem 5.4. Now, it is possible to repeat the part ii) of the proof of Theorem 3.1.

\section{Proof of Theorem 3.3}

For any $d_{2}>0$ we have $\left[d_{1}^{M A X}, d_{2}\right] \in C_{j}$ for a finite number of indices $j>0$ (see Section 2 and also Observation 5.1). Any linear combination $e$ of the eigenfunctions $e_{j}, j>0$ changes the sign (see Lemma $A .1$ for details), therefore the relation $s_{-} e^{-}-s_{+} e^{+} \not \equiv 0$ is always satisfied. Hence, any critical point $\left[d_{1}, d_{2}\right]$ of the problem (8), (4) satisfies $d_{1}<d_{1}^{M A X}$ by Theorem 5.4 . Now, it is possible to repeat the part ii) of the proof of Theorem 3.1.

\section{Proof of Theorem 3.4}

The assumption on $j_{0}$ directly implies that the $j_{0}$-th eigenvalue of the operator $S_{d_{2}}$ in the case $\Gamma_{D} \neq \emptyset$ or of the problem (47a) in the case $\Gamma_{D}=\emptyset$ is positive (see Remarks 5.1, 5.2 and $(17),(18))$. Hence, we have $\left(S_{d_{2}} e_{j_{0}}, e_{j_{0}}\right)>0$, where $e_{j_{0}}$ is the corresponding eigenvector. Let's denote $\tau:=\max \left\{\left\|s_{-}\right\|_{\infty},\left\|s_{+}\right\|_{\infty}\right\}$. We get

$$
\begin{aligned}
\left(S_{d_{2}} e_{j_{0}}, e_{j_{0}}\right)-\left(\beta\left(e_{j_{0}}\right), e_{j_{0}}\right) & =\left(S_{d_{2}} e_{j_{0}}, e_{j_{0}}\right)-\int_{\Omega} s_{+} e_{j_{0}} e_{j_{0}}^{+} d \Omega-\int_{\Omega}-s_{-} e_{j_{0}} e_{j_{0}}^{-} d \Omega \geq \\
& \geq\left(S_{d_{2}} e_{j_{0}}, e_{j_{0}}\right)-\left\|s_{+}\right\|_{\infty} \int_{\Omega}\left(e_{j_{0}}^{+}\right)^{2} d \Omega-\left\|s_{-}\right\|_{\infty} \int_{\Omega}\left(e_{j_{0}}^{-}\right)^{2} d \Omega \\
& \geq\left(S_{d_{2}} e_{j_{0}}, e_{j_{0}}\right)-\tau\left(\int_{\Omega}\left(e_{j_{0}}^{+}\right)^{2} d \Omega+\int_{\Omega}\left(e_{j_{0}}^{-}\right)^{2} d \Omega\right) \geq \\
& \geq\left(S_{d_{2}} e_{j_{0}}, e_{j_{0}}\right)-\tau\left(A e_{j_{0}}, e_{j_{0}}\right) .
\end{aligned}
$$

Since $e_{j_{0}}$ is non-trivial, we have $\left(A e_{j_{0}}, e_{j_{0}}\right)>0$. Hence, if $\tau<\frac{\left(S_{d_{2}} e_{j_{0}}, e_{j_{0}}\right)}{\left(A e_{j_{0}}, e_{j_{0}}\right)}$, then $\left(S_{d_{2}} e_{j_{0}}, e_{j_{0}}\right)-$ $\left(\beta\left(e_{j_{0}}\right), e_{j_{0}}\right)>0$.

If $\Gamma_{D} \neq \emptyset$ then we get

$$
\frac{\left(S_{d_{2}} e_{j_{0}}, e_{j_{0}}\right)}{\left(A e_{j_{0}}, e_{j_{0}}\right)}=\frac{\frac{1}{\kappa_{j_{0}}}\left(\frac{b_{1,2} b_{2,1}}{d_{2} \kappa_{j_{0}}-b_{2,2}}+b_{1,1}\right)\left\|e_{j_{0}}\right\|_{H_{D}^{1}}^{2}}{\frac{1}{\kappa_{j_{0}}}\left\|e_{j_{0}}\right\|_{H_{D}^{1}}^{2}}=\frac{b_{1,2} b_{2,1}}{d_{2} \kappa_{j_{0}}-b_{2,2}}+b_{1,1}
$$

(see Remarks 5.1 and 4.1) and if $\Gamma_{D}=\emptyset$ we get

$$
\frac{\left(S_{d_{2}} e_{j_{0}}, e_{j_{0}}\right)}{\left(A e_{j_{0}}, e_{j_{0}}\right)}=\frac{\frac{1}{\kappa_{j_{0}}+1}\left(\frac{b_{1,2} b_{2,1}}{d_{2} \kappa_{j_{0}}-b_{2,2}}+b_{1,1}\right)\left\|e_{j_{0}}\right\|_{H_{D}^{1}}^{2}}{\frac{1}{\kappa_{j_{0}}+1}\left\|e_{j_{0}}\right\|_{H_{D}^{1}}^{2}}=\frac{b_{1,2} b_{2,1}}{d_{2} \kappa_{j_{0}}-b_{2,2}}+b_{1,1} .
$$

(see Remarks 5.2 and 4.1). Hence, if $\tau<\frac{b_{1,2} b_{2,1}}{d_{2} \kappa_{j_{0}}-b_{2,2}}+b_{1,1}$, then the assumption (53) of Theorems $5.2,5.3$ is satisfied with $\varphi=e_{j_{0}}$ and therefore $d_{1}^{M A X, \beta}>0$ exists. A point $\left[d_{1}^{M A X, \beta}, d_{2}\right]$ is a critical point of (8), (4) by Lemma 5.1 and it lies in $D_{U} \cup C_{E}$ by Theorem 5.4 and because $\left[d_{1}^{M A X}, d_{2}\right] \in C_{E}$ (see Observation 5.1). 


\section{Proof of Theorem 3.5}

If unilateral terms in boundary conditions are considered, we replace the operators $\tilde{F}$ and $\beta$ in $(36),(37),(38),(39)$ by the operator $\beta_{N}$, which has the same properties as $\beta$. Then it is necessary to repeat whole Section 5 for this operator. The actual proof of Theorem 3.5 is then the same as the proof of Theorem 3.1.

\section{A. Appendix}

For a completeness we give here proofs of two standard assertions used in the text (Lemmas A.1, A.2), and a slightly simplified proof of a result given already in [6] (Lemma A.3).

Lemma A.1. Any linear combination $\sum_{k=j_{0}}^{n} a_{k} e_{k}, n \in \mathbb{N}$ of eigenfunctions of (16), where $j_{0}=2$ for $\Gamma_{D} \neq \emptyset$ and $j_{0}=1$ for $\Gamma_{D}=\emptyset$, changes the sign on the domain $\Omega$.

Proof.

Case $\Gamma_{D}=\emptyset$ :

Since $e_{k}$ is an orthonormal basis (see Remark 4.1) and $e_{0}$ is constant, we have

$\int_{\Omega} e_{0} \sum_{k=j_{0}}^{n} a_{k} e_{k} d \Omega=\sum_{k=j_{0}}^{n} a_{k} \int_{\Omega} e_{0} e_{k} d \Omega=\sum_{k=j_{0}}^{n} a_{k} \int_{\Omega}\left(\nabla e_{0} \nabla e_{k}+e_{0} e_{k}\right) d \Omega=\sum_{k=j_{0}}^{n} a_{k}\left(e_{0}, e_{k}\right)_{W^{1,2}}=0$.

Hence, the function $e_{0} \sum_{k=j_{0}}^{n} a_{k} e_{k}$ changes the sign on the domain $\Omega$. Since $e_{0}$ is constant, also the function $\sum_{k=j_{0}}^{n} a_{k} e_{k}$ changes the sign on $\Omega$.

Case $\Gamma_{D} \neq \emptyset$ :

We will use the eigenfunction $e_{1}$ instead of $e_{0}$. Again since $e_{k}$ is the orthonormal basis, we have

$$
\int_{\Omega} e_{1} e_{k} d \Omega=\int_{\Omega}-\frac{1}{\kappa_{1}} \Delta e_{1} e_{k} d \Omega=\frac{1}{\kappa_{1}} \int_{\Omega} \nabla e_{1} \nabla e_{k} d \Omega=\frac{1}{\kappa_{1}}\left(e_{1}, e_{k}\right)_{H_{D}^{1}}=0 \quad \text { for any } k>1 .
$$

The rest is the same as in the case $\Gamma_{D}=\emptyset$.

Lemma A.2. Every bifurcation point $\left[d_{1}, d_{2}\right]$ of $(5),(4)$ is also a critical point of (8), (4).

Proof.

We will show the proof for $\Gamma_{D} \neq \emptyset$. The proof for $\Gamma_{D}=\emptyset$ is the same, we only use the system (39) instead of the system (37).

Let $d^{0}=\left[d_{1}, d_{2}\right] \in \mathbb{R}_{+}^{2}$ be a bifurcation point of (5),(4). Then there exists a sequence $d^{n}=$ $\left[d_{1}^{n}, d_{2}^{n}\right]$ such that $d^{n} \rightarrow d^{0}$ and $W_{n}=\left[u_{n}, v_{n}\right] \rightarrow 0$ with $\left\|W_{n}\right\|=\|u\|_{H_{D}^{1}}+\|v\|_{H_{D}^{1}} \neq 0$ and $d^{n}, W_{n}$ satisfy (5), (4), i.e. (37). We can assume $\frac{W_{n}}{\left\|W_{n}\right\|} \rightarrow W=[w, z]$. Let's divide the system (37) by $\left\|W_{n}\right\|$. We get

$$
\begin{array}{r}
d_{1}^{n} \frac{u_{n}}{\left\|W_{n}\right\|}-b_{1,1} A \frac{u_{n}}{\left\|W_{n}\right\|}-b_{1,2} A \frac{v_{n}}{\left\|W_{n}\right\|}-\frac{N_{1}\left(u_{n}, v_{n}\right)}{\left\|W_{n}\right\|}+\frac{\tilde{F}\left(u_{n}\right)}{\left\|W_{n}\right\|}=0, \\
d_{2}^{n} \frac{v_{n}}{\left\|W_{n}\right\|}-b_{2,1} A \frac{u_{n}}{\left\|W_{n}\right\|}-b_{2,2} A \frac{v_{n}}{\left\|W_{n}\right\|}-\frac{N_{2}\left(u_{n}, v_{n}\right)}{\left\|W_{n}\right\|}=0
\end{array}
$$

due to linearity of $A$. Due to (24) we have $\frac{N_{j}\left(u_{n}, v_{n}\right)}{\left\|W_{n}\right\|} \rightarrow 0$ as $n \rightarrow+\infty$ for $j=1,2$. Since $\frac{u_{n}}{\left\|W_{n}\right\|} \rightarrow w$ and $\frac{v_{n}}{\left\|W_{n}\right\|} \rightarrow z$, using compactness of $A$ and (32) we get $A \frac{u_{n}}{\left\|W_{n}\right\|} \rightarrow A w$ and $\frac{\tilde{F}\left(u_{n}\right)}{\left\|W_{n}\right\|} \rightarrow \beta(w)$, analogously for $v_{n}$ and $z$. We have $d_{1}, d_{2}>0$, therefore it follows from (64) that $\frac{u_{n}}{\left\|W_{n}\right\|} \rightarrow$ $w, \frac{v_{n}}{\left\|W_{n}\right\|} \rightarrow z,\|W\|=1$ and

$$
\begin{aligned}
d_{1}^{0} w-b_{1,1} A w-b_{1,2} A z+\beta(w) & =0 \\
d_{2}^{0} z-b_{2,1} A w-b_{2,2} A z & =0 .
\end{aligned}
$$

Therefore the point $d^{0}$ is a critical point of the system (8), (4). 
Lemma A.3 (see [6]). For any $d_{2}>0$ and $u_{0} \in H_{D}^{1}$ the operators $P \equiv \beta$ and $P \equiv S_{d_{2}}-\beta$ satisfy the condition (51).

Proof.

We will prove (51) for $P=\beta^{-}$. The proof for $\beta^{+}$is analogous and for $P \equiv S_{d_{2}}-\beta$ it will follow by using the definition of $\beta$ and linearity of $S_{d_{2}}$.

Let $u_{0}, h \in H_{D}^{1}$. We will introduce two sets $\Omega_{0}$ and $\Omega_{t h}$ such that

$$
\begin{aligned}
u_{0}(\mathbf{x})<0 \text { a.e. on } \Omega_{0}, & u_{0}(\mathbf{x}) \geq 0 \text { a.e. on } \Omega \backslash \Omega_{0}, \\
u_{0}(\mathbf{x})+t h(\mathbf{x})<0 \text { a.e. on } \Omega_{t h}, & u_{0}(\mathbf{x})+t h(\mathbf{x}) \geq 0 \text { a.e. on } \Omega \backslash \Omega_{t h} .
\end{aligned}
$$

Then

$$
\begin{aligned}
\frac{1}{t}\left(\beta^{-}\left(u_{0}+t h\right)-\beta^{-}\left(u_{0}\right), u_{0}\right) & -\left(\beta^{-}\left(u_{0}\right), h\right)= \\
& =\frac{1}{t}\left[\int_{\Omega}-\left(u_{0}+t h\right)^{-} u_{0}+u_{0} u_{0}^{-} d \Omega\right]-\int_{\Omega}-u_{0}^{-} h d \Omega= \\
& =\frac{1}{t}\left[\int_{\Omega_{t h}}\left(u_{0}+t h\right) u_{0} d \Omega_{t h}-\int_{\Omega_{0}} u_{0}^{2} d \Omega_{0}\right]-\int_{\Omega_{0}} u_{0} h d \Omega_{0}= \\
& =\frac{1}{t}\left[\int_{\Omega_{t h}} u_{0}^{2} d \Omega_{t h}-\int_{\Omega_{0}} u_{0}^{2} d \Omega_{0}\right]+\int_{\Omega_{t h}} u_{0} h d \Omega_{t h}-\int_{\Omega_{0}} u_{0} h d \Omega_{0} .
\end{aligned}
$$

We can afford to work with the definition of $\beta^{-}$without $s_{-}$, because it is non-negative, i.e. it does not affect the sign of terms under integration.

Let $\chi_{t h}$ and $\chi_{0}$ be the characteristic function of $\Omega_{t h}$ and $\Omega_{0}$, respectively. We have

$$
\lim _{t \rightarrow 0} \int_{\Omega_{t h}} u_{0} h d \Omega_{t h}=\lim _{t \rightarrow 0} \int_{\Omega} u_{0} h \chi_{t h} d \Omega=\int_{\Omega} u_{0} h \chi_{0} d \Omega=\int_{\Omega_{0}} u_{0} h d \Omega_{0}
$$

by Dominated Convergence theorem. Let's introduce sets $\Omega_{t h 1}, \Omega_{t h 2}, \Omega_{t h 3}$ such that

$$
\begin{aligned}
& u_{0}(\mathbf{x})<-t h(\mathbf{x}) \text { and } u_{0}(\mathbf{x})<0 \text { almost everywhere on } \Omega_{t h 1}, \\
& u_{0}(\mathbf{x})<-t h(\mathbf{x}) \text { and } u_{0}(\mathbf{x}) \geq 0 \text { almost everywhere on } \Omega_{t h 2}, \\
& u_{0}(\mathbf{x}) \geq-t h(\mathbf{x}) \text { and } u_{0}(\mathbf{x})<0 \text { almost everywhere on } \Omega_{t h 3},
\end{aligned}
$$

with $\Omega_{t h}=\Omega_{t h 1} \cup \Omega_{t h 2}$ and $\Omega_{0}=\Omega_{t h 1} \cup \Omega_{t h 3}$. This way we get

$$
\begin{aligned}
\int_{\Omega_{t h}} u_{0}^{2} d \Omega_{t h}-\int_{\Omega_{0}} u_{0}^{2} d \Omega_{0} & =\int_{\Omega_{t h 1}} u_{0}^{2} d \Omega_{t h 1}+\int_{\Omega_{t h 2}} u_{0}^{2} d \Omega_{t h 2}-\int_{\Omega_{t h 1}} u_{0}^{2} d \Omega_{t h 1}-\int_{\Omega_{t h 3}} u_{0}^{2} d \Omega_{t h 3}= \\
& =\int_{\Omega_{t h 2}} u_{0}^{2} d \Omega_{t h 2}-\int_{\Omega_{t h 3}} u_{0}^{2} d \Omega_{t h 3} .
\end{aligned}
$$

Since $0 \leq u_{0}<-t h$ a.e. on $\Omega_{t h 2}$ and $0>u_{0} \geq-t h$ a.e. on $\Omega_{t h 3}$, we get

$$
\lim _{t \rightarrow 0} \frac{1}{t}\left(\int_{\Omega_{t h 2}} u_{0}^{2} d \Omega_{t h 2}-\int_{\Omega_{t h 3}} u_{0}^{2} d \Omega_{t h 3}\right) \leq \lim _{t \rightarrow 0} \frac{1}{t}\left(\int_{\Omega_{t h 2}}(t h)^{2} d \Omega_{t h 2}-\int_{\Omega_{t h 3}}(t h)^{2} d \Omega_{t h 3}\right)=0 .
$$

Hence, it follows from the discussion above that

$$
\lim _{t \rightarrow 0} \frac{1}{t}\left(\beta^{-}\left(u_{0}+t h\right)-\beta^{-}\left(u_{0}\right), u_{0}\right)-\left(\beta^{-}\left(u_{0}\right), h\right)=0
$$

which proves (51) for $\beta^{-}$.

\section{Acknowledgement}

M.Fencl has been supported by the project SGS-2016-003 of University of West Bohemia and the project LO1506 of the Czech Ministry of Education, Youth and Sport. M. Kučera has been supported by RVO:67985840. 


\section{Bibliography}

\section{References}

[1] M. A. J. CHAPLAIN, M. GANESH, and I. G. GRAHAM, Spatio-temporal pattern formation on spherical surfaces: numerical simulation and application to solid tumour growth, J. Math. Biol., 42 (2001), pp. 387-423.

[2] J. EISNER AND M. KUČERA, Spatial patterns for reaction-diffusion systems with conditions described by inclusions, Appl. Math., 42 (1997), pp. 421-449.

[3] J. EISNER AND M. KUČERA, Bifurcation of solutions to reaction-diffusion systems with jumping nonlinearities, in Applied Nonlinear Analysis, edited by A. Sequeira, H. Beirao da Veiga and J. H. Videman, Kluwer Academic/Plenum Publishers, New York 1999, pp. 79-96

[4] J. EISNER, M. KUČERA, AND M. VÄTH, Degree and global bifurcation for elliptic equations with multivalued unilateral conditions, Nonlinear Anal., 64 (2006), pp. 1710-1736.

[5] M. KUČERA, Reaction-diffusion systems: stabilizing effect of conditions described by quasivariational inequalities, Czechoslovak Math. J., 47(122) (1997), pp. 469-486.

[6] M. KUČERA AND J. NAVRÁTIL, Eigenvalues and bifurcation for problems with positively homogeneous operators and reaction-diffusion systems with unilateral terms, Nonlinear Anal., 166 (2018), pp. 154-180.

[7] M. KUČERA AND M. VÄTH, Bifurcation for a reaction-diffusion system with unilateral and Neumann boundary conditions, J. Differential Equations, 252 (2012), pp. 2951-2982.

[8] M. MIMURA, Y. NISHIURA, AND M. YAMAGUTI, Some diffusive prey and predator systems and their bifurcation problems, Ann. N. Y. Acad. Sci. 316 (1979), 490-510.

[9] J. D. MURRAY, Mathematical Biology II: Spatial Models and Biomedical Applications, Interdisciplinary Applied Mathematics, vol. 18, Springer-Verlag, New York, third ed., 2003.

[10] Y. NISHIURA, Global structure of bifurcating solutions of some reaction-diffusion systems, SIAM J. Math. Anal., 13 (1982), pp. 555-593.

[11] A. M. TURING, The chemical basis of morphogenesis, Philos. Trans. Roy. Soc. London Ser. B, 237 (1952), pp. 37-72.

[12] T. VEJCHODSKÝ, F. JAROŠ, M. KUČERA, AND V. RYBÁr̆, Unilateral regulation breaks regularity of Turing patterns, Phys. Rev. E, 96022212 (2017).

[13] W. P. ZIEMER, Weakly Differentiable Functions: Sobolev Spaces and Functions of Bounded Variation, Graduate Texts in Mathematics, vol. 120, Springer-Verlag, New York, 1989. 